\title{
Stability of Cascaded Fuzzy Systems and Observers
}

\author{
Zsófia Lendek, Robert Babuška, and Bart De Schutter, Member, IEEE
}

\begin{abstract}
A large class of nonlinear systems can be well approximated by Takagi-Sugeno (TS) fuzzy models with linear or affine consequents. It is well known that the stability of these consequent models does not ensure the stability of the overall fuzzy system. Therefore, several stability conditions have been developed for TS fuzzy systems. We study a special class of nonlinear dynamic systems that can be decomposed into cascaded subsystems, which are represented as TS fuzzy models. We analyze the stability of the overall TS system based on the stability of the subsystems and prove that the stability of the subsystems implies the stability of the overall system. The main benefit of this approach is that it relaxes the conditions imposed when the system is globally analyzed, thereby solving some of the feasibility problems. Another benefit is that by using this approach, the dimension of the associated linear matrix inequality (LMI) problem can be reduced. For naturally distributed applications, such as multiagent systems, the construction and tuning of a centralized observer may not be feasible. Therefore, we also extend the cascaded approach to the observer design and use fuzzy observers to individually estimate the states of these subsystems. A theoretical proof of stability and simulation examples are presented. The results show that the distributed observer achieves the same performance as the centralized one, while leading to increased modularity, reduced complexity, lower computational costs, and easier tuning. Applications of such cascaded systems include multiagent systems, distributed process control, and hierarchical large-scale systems.
\end{abstract}

Index Terms-Cascaded systems, fuzzy observers, fuzzy systems, Lyapunov stability.

\section{INTRODUCTION}

$\mathbf{M}$ ANY PROBLEMS in decision making, control, and monitoring require the estimation of states and possibly uncertain parameters, based on a dynamic system model and a sequence of noisy measurements. For such a purpose, dynamic systems are often modeled in the state-space framework, using the state-transition model, which describes the evolution of states over time, and the sensor model, which relates the measurements to the states.

Traditionally, the class of linear, time-invariant systems has dominated control theory. The linearity and time-invariance make this type of system easy to analyze. The disadvantages are that such systems fail to describe nonlinear systems globally.

Manuscript received April 23, 2007; revised September 25, 2007; accepted December 28, 2007. First published April 30, 2008; current version published June 11, 2009. This work was supported by Senter, Ministry of Economic Affairs of the Netherlands, within the project Interactive Collaborative Information Systems, under Grant BSIK03024.

Zs. Lendek and R. Babuška are with the Delft Center for Systems and Control, Delft University of Technology, 2628 CD Delft, The Netherlands (e-mail: z.lendek@tudelft.nl; r.babuska@tudelft.nl).

B. De Schutter is with the Delft Center for Systems and Control and the Marine and Transport Technology Department, Delft University of Technology, 2628 CD Delft, The Netherlands (e-mail: b@deschutter.info).

Color versions of one or more of the figures in this paper are available online at http://ieeexplore.ieee.org.

Digital Object Identifier 10.1109/TFUZZ.2008.924353
An accurate approximation of a nonlinear system can only be expected in the vicinity of an equilibrium point.

A generic method for the design of an observer valid for all types of nonlinear systems has yet to be developed. A large class of nonlinear systems can be well approximated by TakagiSugeno (TS) fuzzy models [1], which, in theory, can approximate a general nonlinear system to an arbitrary degree of accuracy [2]. Stability conditions have been derived for TS fuzzy systems, most of them relying on the feasibility of an associated system of linear matrix inequalities (LMIs) [3]-[5]. A comprehensive survey on the analysis of fuzzy systems can be found in [6].

For a general nonlinear system represented by a fuzzy model, well-established methods and algorithms can be used to design fuzzy observers; therefore, the analysis and design become much easier. Several types of observers have been developed for TS fuzzy systems, which include fuzzy Thau-Luenberger observers [3], [7], reduced order observers [5], [8], and slidingmode observers [9]. In general, the design methods for observers also lead to an LMI feasibility problem. However, the complexity of the system grows exponentially with the number of antecedents, and the stability analysis problem eventually becomes intractable for a large number of rules.

Decentralized state estimation has been studied in the context of large-scale processes and distributed systems. The decentralized architecture generally has the form of a network of sensor nodes, each with its own computing capability. In the case of a fully decentralized system, computations are performed locally, and communication takes place between any two nodes. Each node shares information with other nodes and computes a local estimate. Computation and communication is distributed over the network, and the global estimate is computed by fusing the local results. Several topologies have been proposed, depending on the particular application. In case of large-scale processes [10], [11], the network is generally in a hierarchical form, with several intermediate nodes and one final fusion node. For distributed systems, such as multiagent societies [12]-[14], several fusion nodes are used, which process the data and send the information to the rest of the nodes. Observers for distributed estimation include, but are not limited to, the decentralized Kalman and the extended Kalman filter [15], the information filter, and several types of particle filters [16], [17].

An important class of distributed systems can be represented as cascaded subsystems (e.g., material processing systems, chemical processes). In several cases, conclusions referring to the overall system can be drawn based on the study of the individual subsystems. For instance, for linear systems, the stability of the subsystems implies the stability of the cascaded system [18]. However, this property in general does not hold for nonlinear or time-varying systems. Even global asymptotic 
stability of the individual subsystems does not necessarily imply stability of the cascade.

In the literature, the stability of several types of cascaded systems has been studied. The main motivation came from the linear-nonlinear cascade [19], resulting from input-output linearization. Conditions to ensure the overall stability of more general cascades, in which both subsystems are nonlinear, were derived in [18], [20], and [21].

The contribution of this paper is twofold. First, we study a special class of systems, which are represented as TS fuzzy systems, which can be decomposed into cascaded subsystems, and analyze the stability of the whole system based on the stability of the subsystems. The idea behind this type of stability analysis is that many systems are naturally distributed (e.g., traffic control systems) or cascaded (e.g., hierarchical largescale systems), while others may be represented as cascaded, observable subsystems, which are less complex than the original system. The main benefit of this approach is that it relaxes the conditions imposed by analyzing the system globally. Global analysis may lead to infeasible LMI conditions, even if the system is stable. We propose relaxed stability conditions, which may render the associated LMI problem feasible. Moreover, the dimension of the associated LMI problem is generally reduced.

Second, the results are extended to observer design. We analyze the joint performance of fuzzy observers individually designed for the subsystems. The benefit of this type of estimation is that separate observers can be designed for the individual subsystems, which makes their tuning easier. Moreover, different types of observers can be combined, depending on the subsystem considered. Such a cascaded system can be regarded as a cooperative multiagent system, where each agent observes at least its own states and makes decisions based on these observations. The agents rely on their own measurements and the information gathered from other agents. In turn, each agent communicates its own results to other agents. If all the agents in a system use the same observer method, then such an observer system can be designed and implemented in a modular manner, i.e., after identifying the coupling among the subsystems, the observers can be designed in a similar fashion. We present a theoretical comparison of the centralized and cascaded fuzzy observers and compare their performance on two examples.

The structure of the paper is as follows. Section II introduces the cascaded setting for nonlinear systems and gives stability conditions for such systems. Section III reviews stability conditions for TS fuzzy systems and observers. The proposed stability conditions for cascaded fuzzy systems and observers are presented in Section IV. Examples are given in Section V. Finally, Section VI concludes the paper.

\section{Stability OF CASCADED DynAmic Systems}

In the literature, the main motivation to consider cascaded dynamical systems came from the analysis of the models obtained after input-output linearization [18], [19]. Several stability conditions were derived for different types of subsystems. In this section, the cascaded setting for general nonlinear systems

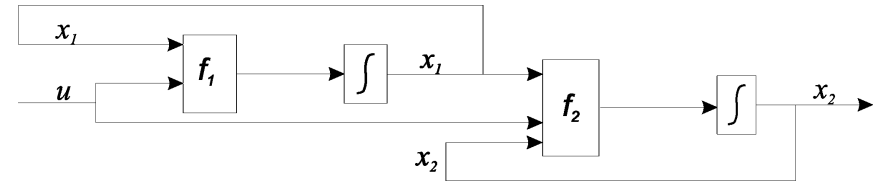

Fig. 1. Cascaded subsystems.

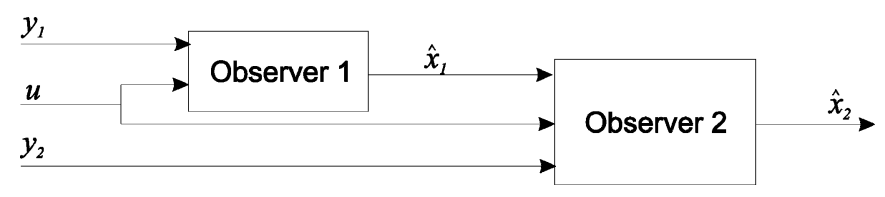

Fig. 2. Cascaded observers.

and observers is presented, together with the relevant stability conditions.

\section{A. Preliminaries}

Consider the following general, observable nonlinear system:

$$
\begin{array}{cc}
\dot{x}_{1}=f_{1}(\boldsymbol{x}, \boldsymbol{u}), & y_{1}=h_{1}(\boldsymbol{x}, \boldsymbol{u}) \\
\dot{x}_{2}=f_{2}(\boldsymbol{x}, \boldsymbol{u}), & y_{2}=h_{2}(\boldsymbol{x}, \boldsymbol{u}) \\
\vdots & \vdots \\
\dot{x}_{n}=f_{n}(\boldsymbol{x}, \boldsymbol{u}), & y_{m}=h_{m}(\boldsymbol{x}, \boldsymbol{u})
\end{array}
$$

where $\boldsymbol{x}=\left[x_{1}, \ldots, x_{n}\right]^{T}$ and $\boldsymbol{u}=\left[u_{1}, \ldots, u_{l}\right]^{T}$, and assume that this system can be partitioned into subsystems. For the ease of notation, only two subsystems are considered, without loss of generality:

$$
\begin{aligned}
\dot{\boldsymbol{x}}_{1} & =\boldsymbol{f}_{1}\left(\boldsymbol{x}_{1}, \boldsymbol{u}\right) \\
\boldsymbol{y}_{1} & =\boldsymbol{h}_{1}\left(\boldsymbol{x}_{1}, \boldsymbol{u}\right)
\end{aligned}
$$

and

$$
\begin{array}{r}
\dot{\boldsymbol{x}}_{2}=\boldsymbol{f}_{2}\left(\boldsymbol{x}_{1}, \boldsymbol{x}_{2}, \boldsymbol{u}\right) \\
\boldsymbol{y}_{2}=\boldsymbol{h}_{2}\left(\boldsymbol{x}_{1}, \boldsymbol{x}_{2}, \boldsymbol{u}\right)
\end{array}
$$

where $\boldsymbol{x}=\left[\begin{array}{ll}\boldsymbol{x}_{1}^{T} & \boldsymbol{x}_{2}^{T}\end{array}\right]^{T}, \boldsymbol{y}=\left[\begin{array}{ll}\boldsymbol{y}_{1}^{T} & \boldsymbol{y}_{2}^{T}\end{array}\right]^{T}$ (with possible reordering) so that (2) is observable. Note that since both the systems (1) and (2) are observable, subsystem (3) is also observable. In fact, for subsystem (3), $\boldsymbol{x}_{1}$ is an input. In general, such a partition of the model does not necessarily exist. Moreover, if a partition exists, it might not be unique.

Given a particular nonlinear system of the form (1), with at least two measurements, a partitioning into two subsystems can be constructed easily, while also deciding whether the partitioning is possible. For two subsystems, the cascaded structure is depicted in Fig. 1.

If such a partition exists, observers may be designed for the subsystems separately, with some observers using the estimates obtained by other observers. For two subsystems, the cascaded observer structure is depicted in Fig. 2. 


\section{B. Stability of Cascaded Systems}

It is well known that the cascade of stable linear systems is stable, since the eigenvalues of the joint system are determined only by the eigenvalues of the individual subsystems [18]. Therefore, the stability of the joint system is determined by the stability of the subsystems. However, the same reasoning does not necessarily hold for nonlinear or time-varying systems. Even the global asymptotic stability of the decoupled subsystems does not necessarily imply the stability of the cascade.

In the literature, the stability of several special cases has been studied. The main motivation comes from the linear-nonlinear cascade [19], resulting from input-output linearization. More general cascades, in which both subsystems are nonlinear, were studied, and conditions to ensure overall stability were derived in [18]. A selection of relevant results is presented next.

Definition 1: A continuous function $\alpha: \mathcal{R}^{+} \rightarrow \mathcal{R}^{+}$belongs to class $\mathcal{K}$ if it is strictly increasing and $\alpha(0)=0$. If $\alpha(s) \rightarrow \infty$ when $s \rightarrow \infty$, then $\alpha$ is said to be of class $\mathcal{K}_{\infty}$.

Definition 2: A system $\dot{\boldsymbol{x}}=\boldsymbol{f}(\boldsymbol{x}, \boldsymbol{u})$ is input-to-state stable (ISS) if and only if there exist a positive definite proper function $V(\boldsymbol{x})$ and two class $\mathcal{K}$ functions $\alpha_{1}$ and $\alpha_{2}$ such that

$$
\left\{\|\boldsymbol{x}\| \geq \alpha_{1}(\|\boldsymbol{u}\|)\right\} \Rightarrow\left\{\frac{\partial V(\boldsymbol{x})}{\partial \boldsymbol{x}} \boldsymbol{f}(\boldsymbol{x}, \boldsymbol{u}) \leq-\alpha_{2}(\|\boldsymbol{x}\|)\right\}
$$

where $\|\cdot\|$ represents the Euclidian norm.

Consider the nonlinear, cascaded, autonomous system

$$
\begin{aligned}
& \dot{\boldsymbol{x}}_{1}=\boldsymbol{f}_{1}\left(\boldsymbol{x}_{1}\right) \\
& \dot{\boldsymbol{x}}_{2}=\boldsymbol{f}_{2}\left(\boldsymbol{x}_{1}, \boldsymbol{x}_{2}\right) .
\end{aligned}
$$

It has been shown in [22] that if

1) the functions $f_{1}$ and $f_{2}$ are sufficiently smooth in their arguments,

2) system (6) is input-to-state-stable with regard to the input $\boldsymbol{x}_{1}$, and

3) system (5) and the system

$$
\dot{\boldsymbol{x}}_{2}=\boldsymbol{f}_{2}\left(0, \boldsymbol{x}_{2}\right)
$$

are globally asymptotically stable (GAS)

then the cascade (5) and (6) is GAS. An equivalent sufficient stability condition is presented in [21]; the cascaded system is GAS if both subsystems are GAS and all solutions are bounded. The main difficulty with this approach is that, in general, boundedness of all the solutions is not easy to determine, and the conditions to ensure boundedness may be very conservative.

More relaxed sufficient stability conditions have been derived for systems of the form

$$
\begin{aligned}
& \dot{\boldsymbol{x}}_{1}=\boldsymbol{f}_{1}\left(\boldsymbol{x}_{1}\right) \\
& \dot{\boldsymbol{x}}_{2}=\boldsymbol{f}_{2}\left(\boldsymbol{x}_{2}\right)+\boldsymbol{g}\left(\boldsymbol{x}_{1}, \boldsymbol{x}_{2}\right)
\end{aligned}
$$

assuming that the individual subsystems are GAS, and additionally, certain restrictions related to the continuity and/or slope apply for the interconnection term $\boldsymbol{g}$ [19], [23], [24]. A theorem for ensuring that the cascaded system (8) is uniformly GAS (UGAS) [18] is presented next.

\section{Assumptions:}

1) System (7) is UGAS.

2) There exist constants $c_{1}, c_{2}, \mu>0$ and a Lyapunov function $V\left(t, \boldsymbol{x}_{2}\right)$ for (7) such that $V: \mathcal{R}^{+} \times \mathcal{R}^{n} \rightarrow \mathcal{R}^{+}$is positive definite, radially unbounded, $\dot{V}\left(t, \boldsymbol{x}_{2}\right) \leq 0$, and

$$
\begin{array}{ll}
\left\|\frac{\partial V}{\partial \boldsymbol{x}_{2}}\right\|\left\|\boldsymbol{x}_{2}\right\| \leq c_{1} V\left(t, \boldsymbol{x}_{2}\right) & \forall \boldsymbol{x}_{2}:\left\|\boldsymbol{x}_{2}\right\|>\mu \\
\left\|\frac{\partial V}{\partial \boldsymbol{x}_{2}}\right\| \leq c_{2} & \forall \boldsymbol{x}_{2}:\left\|\boldsymbol{x}_{2}\right\| \leq \mu .
\end{array}
$$

3) There exist two continuous functions $\theta_{1}, \theta_{2}: \mathcal{R}^{+} \rightarrow \mathcal{R}^{+}$ such that $\boldsymbol{g}(\boldsymbol{x})$ satisfies

$$
\|\boldsymbol{g}(\boldsymbol{x})\| \leq \theta_{1}\left(\left\|\boldsymbol{x}_{1}\right\|\right)+\theta_{2}\left(\left\|\boldsymbol{x}_{1}\right\|\right)\left\|\boldsymbol{x}_{2}\right\| .
$$

4) There exists a class $\mathcal{K}$ function $\alpha(\cdot)$ so that for all $t_{0} \geq 0$, the trajectories of the system (5) satisfy

$$
\int_{t_{0}}^{\infty}\left\|\boldsymbol{x}_{1}\left(t ; t_{0}, \boldsymbol{x}_{1}\left(t_{0}\right)\right)\right\| d t \leq \alpha\left(\left\|\boldsymbol{x}_{1}\left(t_{0}\right)\right\|\right) .
$$

Theorem 1: Let Assumption 1 hold and suppose that the trajectories of (5) are uniformly globally bounded. If, in addition, Assumptions 2-4 are satisfied, then the solutions of system (8) are uniformly globally bounded. If furthermore, system (5) is UGAS, then so is the cascaded system (8).

Proposition 1: If, in addition to the aforementioned assumptions, systems (5) and (7) are exponentially stable, then the cascaded system (8) is also exponentially stable.

The proof of Theorem 1, Proposition 1, and the study of different cases of interconnection terms can be found in [18] and [19], and stabilizability conditions were derived in [24] and [25]. For observer design for a special type of cascaded single-input single-output (SISO) system, see [26].

\section{STABILITY OF FUZZY SYSTEMS}

Though the consequents of TS fuzzy systems are usually chosen to be linear or affine, it is well known that the stability of these local models does not imply the stability of the overall fuzzy system. During operation of the full fuzzy model, the local models are blended. The particular blending of several local models may be strongly nonlinear, which influences the stability. The switching surfaces between the models depend on the operators used for intersection, union, and implication in the antecedent of the rules. Several such operators can be used. Some of them are continuous like the product or sum; others, however, are not (e.g., MIN and MAX). MIN and MAX operators cause rapid switching in fuzzy models, and therefore, the model surface is not smooth. The smoothness of the premise membership functions influences the smoothness of the switching of the local models and, consequently, the general stability of the system. It is theoretically a very difficult problem to establish the stability of a fuzzy system considering the possible switching surfaces, and therefore, several stability conditions were derived that ensure the stability of the system for any normalized membership functions, independent of the operators used in the antecedent rules. Most of these conditions depend on the feasibility of an associated LMI problem. Some of them 
are reviewed later. Throughout the paper it is assumed that the membership functions are normalized.

\section{A. Autonomous Fuzzy Systems}

Consider the autonomous fuzzy system expressed as

$$
\dot{\boldsymbol{x}}=\sum_{i=1}^{m} w_{i}(\boldsymbol{z}) A_{i} \boldsymbol{x}
$$

where $A_{i}, i=1,2, \ldots, m$ represents the $i$ th local linear model, $w_{i}$ is the corresponding normalized membership function, and $z$ the vector of the scheduling parameters. System (12) can also be written as

$$
\dot{\boldsymbol{x}}=A(\boldsymbol{z}) \boldsymbol{x}
$$

with $A(\boldsymbol{z})=\sum_{i=1}^{m} w_{i}(\boldsymbol{z}) A_{i}$.

For system (12), several stability conditions were derived. Among them, a well-known and frequently used condition is now formulated [3].

Theorem 2: System (12) is exponentially stable if there exists $P=P^{T}>0$ so that $A_{i}^{T} P+P A_{i}^{T}<0$, for $i=1,2, \ldots, m$. $\square$

A condition on the convergence rate of system (12) was also derived from Theorem 2 [3].

Theorem 3: The decay rate of system (12) is at least $\alpha$, if there exists $P=P^{T}>0$ so that

$$
A_{i}^{T} P+P A_{i}+2 \alpha P<0, \quad i=1,2, \ldots, m .
$$

Stability conditions similar to those of Theorem 2 can be used if the system considered is subjected to vanishing disturbances. Consider the following perturbed fuzzy system:

$$
\dot{\boldsymbol{x}}=\sum_{i=1}^{m} w_{i}(\boldsymbol{z}) A_{i} \boldsymbol{x}+D \boldsymbol{f}(t, \boldsymbol{x})
$$

where $D$ is a perturbation distribution matrix and $\boldsymbol{f}$ is a vanishing disturbance, i.e., $\boldsymbol{f}(t, \boldsymbol{x}) \rightarrow 0$ when $t \rightarrow \infty$, and assume that $\boldsymbol{f}$ is Lipschitz, i.e., there exists $\mu>0$ so that $\|\boldsymbol{f}(t, \boldsymbol{x})\| \leq \mu\|\boldsymbol{x}\|$ for all $t$ and $\boldsymbol{x}$. With these assumptions, a sufficient stability condition can be formalized by the following theorem [27].

Theorem 4: System (14) is exponentially stable if there exist matrices $P=P^{T}, Q=Q^{T}$ so that

$$
\begin{aligned}
& P>0, \quad Q>0 \\
& \mu \leq \frac{\lambda_{\min }(Q)}{\|P D\|} \\
& A_{i}^{T} P+P A_{i}^{T}<-2 Q, \quad i=1,2, \ldots, m
\end{aligned}
$$

where $\lambda_{\min }$ is the eigenvalue with the smallest absolute value.

Several variants of this theorem exist, together with algorithms to compute robustness measures [27]. However, these approaches are conservative by disregarding the fact that the rules are valid only in a region of the state space. For fuzzy systems, the membership functions often have bounded support. Therefore, it is sufficient that $\boldsymbol{x}^{T}\left(A_{i}^{T} P+P A_{i}^{T}\right) \boldsymbol{x}<0$ only where $w_{i}(\boldsymbol{z})>0$. Stability conditions for the case when the support of each membership function can be bounded were derived in [4].
Another approach, which is based on partitioning the state space into operating and interpolation regimes $X_{k}, k=$ $1,2, \ldots, K, K$ being the number of regimes, and $K_{i}$ the index set of the local models active in the regime $i$, is described in [28]. Assuming that in (12), $z$ can be expressed as some function of $\boldsymbol{x}$, the system can be written as

$$
\dot{\boldsymbol{x}}=\sum_{i \in K_{k}} w_{i}(\boldsymbol{x}) A_{i} \boldsymbol{x}, \quad \boldsymbol{x} \in X_{k}
$$

where $K_{k}$ is the index set of the linear subsystems active in the region $X_{k}$. Then, one can use a Lyapunov function $V_{k}(\boldsymbol{x})=\boldsymbol{x}^{T} P_{k} \boldsymbol{x}$ for region $X_{k}$, i.e., the corresponding part of the Lyapunov function $V(\boldsymbol{x})=\sum_{k=1}^{K} w_{k}(\boldsymbol{x}) \boldsymbol{x}^{T} P_{k} \boldsymbol{x}$ for the whole system. The system (16) is stable under the conditions expressed by the following theorem [28].

Theorem 5: The system (16) is GAS if there exist matrices $P_{i}=P_{i}^{T}, H=H^{T}>0, F_{i}, i=1,2, \ldots, K$ so that

$$
\begin{aligned}
& P_{i}=F_{i}^{T} H F_{i} \\
& P_{i}>0 \\
& F_{i} \boldsymbol{x}=F_{j} \boldsymbol{x} \quad \forall \boldsymbol{x} \in X_{i} \cap X_{j} \\
& A_{k}^{T} P_{i}+P_{i} A_{k}<0 \quad \forall k \in K_{i} .
\end{aligned}
$$

For more relaxed conditions and how to compute the corresponding matrices, see [29] and [30]. Similar conditions for the discrete-time case are described in [31].

Note that all the earlier conditions rely on the feasibility of a derived LMI problem. Since efficient algorithms exist for solving LMIs, they can be easily verified. However, two shortcomings of these theorems must be mentioned: 1) The conditions are conservative and often lead to infeasible LMIs, and 2) the number of LMIs associated in particular to the conditions of Theorem 5 is, in the worst case, exponential in the number of local models.

\section{B. Fuzzy Observers}

Consider now the affine fuzzy system

$$
\begin{aligned}
& \dot{\boldsymbol{x}}=\sum_{i=1}^{m} w_{i}(\boldsymbol{z})\left(A_{i} \boldsymbol{x}+B_{i} \boldsymbol{u}+a_{i}\right) \\
& \boldsymbol{y}=\sum_{i=1}^{m} w_{i}(\boldsymbol{z})\left(C_{i} \boldsymbol{x}+d_{i}\right)
\end{aligned}
$$

and an observer of the form

$$
\begin{aligned}
& \dot{\widehat{\boldsymbol{x}}}=\sum_{i=1}^{m} w_{i}(\boldsymbol{z})\left(A_{i} \widehat{\boldsymbol{x}}+B_{i} \boldsymbol{u}+a_{i}+L_{i}(\boldsymbol{y}-\widehat{\boldsymbol{y}})\right) \\
& \widehat{\boldsymbol{y}}=\sum_{i=1}^{m} w_{i}(\boldsymbol{z})\left(C_{i} \widehat{\boldsymbol{x}}+d_{i}\right) .
\end{aligned}
$$

As before, it is assumed that the membership functions are normal. Depending on the explicit form of the error system given by $\dot{\boldsymbol{e}}=\dot{\boldsymbol{x}}-\dot{\widehat{\boldsymbol{x}}}$, the theorems presented in Section III-A can be applied directly, or similar conditions may be derived 
to ensure the stability of the observer. For the analysis, two cases are distinguished: 1) The scheduling vector $z$ does not depend on the estimated states, and 2) $z$ depends on (some of) the estimated states, that is, $\boldsymbol{z}=\widehat{\boldsymbol{z}}$.

1) Scheduling Vector Does Not Depend on the Estimated States: In this case, the error system can be written as

$$
\dot{\boldsymbol{e}}=\sum_{i=1}^{m} \sum_{j=1}^{m} w_{i}(\boldsymbol{z}) w_{j}(\boldsymbol{z})\left(A_{i}-L_{i} C_{j}\right) \boldsymbol{e} .
$$

Using a Lyapunov function of the form $V(t)=\boldsymbol{e}^{T} P \boldsymbol{e}$, with $P=P^{T}>0$, basic sufficient stability conditions for this system were derived in [3].

Theorem 6: The system (20) is GAS if there exists $P=P^{T}>$ 0 so that for $i=1,2, \ldots, m$

$$
\begin{aligned}
& \left(A_{i}-L_{i} C_{i}\right)^{T} P+P\left(A_{i}-L_{i} C_{i}\right)<0 \\
& \left(G_{i j}+G_{j i}\right)^{T} P+P\left(G_{i j}+G_{j i}\right) \leq 0 \\
& G_{i j}=A_{i}-L_{i} C_{j} \\
& \text { for } j=1,2, \ldots, m \text { for } w_{i}(\boldsymbol{z}) w_{j}(\boldsymbol{z})>0 .
\end{aligned}
$$

A well-known condition on the design of the observer for the system (12), so that a desired convergence rate $\alpha$ is guaranteed, is presented next [3].

Theorem 7: The decay rate of the error system (20) is at least $\alpha$ if there exists $P=P^{T}>0$ so that

$$
\begin{aligned}
& \left(A_{i}-L_{i} C_{i}\right)^{T} P+P\left(A_{i}-L_{i} C_{i}\right)+2 \alpha P<0 \\
& i=1,2, \ldots, m \\
& \left(A_{i}-L_{i} C_{j}\right)^{T} P+P\left(A_{i}-L_{i} C_{j}\right)+2 \alpha P<0 \\
& i, j=1,2, \ldots, m \text { for } w_{i}(\boldsymbol{z}) w_{j}(\boldsymbol{z}) \neq 0 .
\end{aligned}
$$

2) Scheduling Vector Depends on the Estimated States: The second case is when the scheduling vector depends on the states to be estimated, i.e., $\boldsymbol{z}=\widehat{\boldsymbol{z}}$. For simplicity, only the case with common measurement matrices will be considered. Then, the observer (19) becomes

$$
\begin{aligned}
& \dot{\widehat{\boldsymbol{x}}}=\sum_{i=1}^{m} w_{i}(\widehat{\boldsymbol{z}})\left(A_{i} \widehat{\boldsymbol{x}}+B_{i} \boldsymbol{u}+a_{i}+L_{i}(\boldsymbol{y}-\widehat{\boldsymbol{y}})\right) \\
& \widehat{\boldsymbol{y}}=C \widehat{\boldsymbol{x}}
\end{aligned}
$$

and the error dynamics can be expressed as

$$
\begin{aligned}
\dot{\boldsymbol{e}}= & \sum_{i=1}^{m} w_{i}(\widehat{\boldsymbol{z}})\left(A_{i}-L_{i} C\right) \boldsymbol{e} \\
& +\sum_{i=1}^{m}\left(w_{i}(\boldsymbol{z})-w_{i}(\widehat{\boldsymbol{z}})\right)\left(A_{i} \boldsymbol{x}+B_{i} \boldsymbol{u}+a_{i}\right) .
\end{aligned}
$$

For such a system, sufficient stability conditions are given by the following theorem [27].

Theorem 8: The error system (22) is exponentially stable, if there exist $\mu>0, P=P^{T}>0, Q=Q^{T}>0$ so that for all

$$
\begin{array}{r}
i=1, \ldots, m \\
\left(A_{i}-L_{i} C\right)^{T} P+P\left(A_{i}-L_{i} C\right) \leq Q \\
\left(\begin{array}{cc}
Q-\mu^{2} & P \\
P & I
\end{array}\right)>0 \\
\left\|\left(w_{i}(\mathbf{z})-w_{i}(\widehat{\mathbf{z}})\right)\left(A_{i} \mathbf{x}+B_{i} \mathbf{u}+a_{i}\right)\right\| \leq \mu\|e\|
\end{array}
$$

i.e., $\left(w_{i}(\boldsymbol{z})-w_{i}(\widehat{\boldsymbol{z}})\right)\left(A_{i} \boldsymbol{x}+B_{i} \boldsymbol{u}+a_{i}\right)$ is bounded by a linear growth of $e$.

\section{StABILITy OF CASCADED FuZZY OBSERVERS}

In this section, stability conditions for cascaded TS fuzzy systems and observers are derived. We prove that, thanks to the special form of the TS fuzzy system, the stability of the individual subsystems implies the stability of the global cascaded system.

\section{A. Cascaded Fuzzy Systems}

Consider the case when the system matrices of the model (12) for each rule $i=1,2, \ldots, m$ can be written as

$$
A_{i}=\left(\begin{array}{cc}
A_{1} & 0 \\
A_{21} & A_{2}
\end{array}\right)_{i}=\left(\begin{array}{cc}
A_{1 i} & 0 \\
A_{21 i} & A_{2 i}
\end{array}\right)
$$

i.e., system (12) can be expressed as the cascade of two fuzzy systems:

$$
\begin{aligned}
& \dot{\boldsymbol{x}}_{1}=\sum_{i=1}^{m} w_{1 i}\left(\boldsymbol{z}_{1}\right) A_{1 i} \boldsymbol{x}_{1} \\
& \dot{\boldsymbol{x}}_{2}=\sum_{i=1}^{m} w_{2 i}\left(\boldsymbol{z}_{1}, \boldsymbol{z}_{2}\right)\left(A_{21 i} \boldsymbol{x}_{1}+A_{2 i} \boldsymbol{x}_{2}\right)
\end{aligned}
$$

or equivalently

$$
\begin{aligned}
& \dot{\boldsymbol{x}}_{1}=A_{1}\left(\boldsymbol{z}_{1}\right) \boldsymbol{x}_{1} \\
& \dot{\boldsymbol{x}}_{2}=A_{21}\left(\boldsymbol{z}_{1}, \boldsymbol{z}_{2}\right) \boldsymbol{x}_{1}+A_{2}\left(\boldsymbol{z}_{1}, \boldsymbol{z}_{2}\right) \boldsymbol{x}_{2}
\end{aligned}
$$

with normalized membership functions $w_{1 i}$ and $w_{2 i}$, $\boldsymbol{x}=\left[\boldsymbol{x}_{1}^{T}, \boldsymbol{x}_{2}^{T}\right]^{T}, \boldsymbol{z}=\left[\boldsymbol{z}_{1}^{T}, \boldsymbol{z}_{2}^{T}\right]^{T}, A_{1}\left(\boldsymbol{z}_{1}\right)=\sum_{i=1}^{m} w_{i}\left(\boldsymbol{z}_{1}\right) A_{1 i}$, $A_{2}(\boldsymbol{z})=\sum_{i=1}^{m} w_{2 i}(\boldsymbol{z}) A_{2 i}$, etc.

Now, we prove that, if the subsystems

$$
\dot{\boldsymbol{x}}_{1}=A_{1}\left(\boldsymbol{z}_{1}\right) \boldsymbol{x}_{1}
$$

and

$$
\dot{\boldsymbol{x}}_{2}=A_{2}\left(\boldsymbol{z}_{1}, \boldsymbol{z}_{2}\right) \boldsymbol{x}_{2}
$$

are UGAS, then it is possible to apply Theorem 1 to fuzzy systems of the form (24).

Theorem 9: If there exist two Lyapunov functions of the form $V_{1}\left(\boldsymbol{x}_{1}\right)=\boldsymbol{x}_{1}^{T} P_{1} \boldsymbol{x}_{1}$ and $V_{2}\left(\boldsymbol{x}_{2}\right)=\boldsymbol{x}_{2}^{T} P_{2} \boldsymbol{x}_{2}$ so that the subsystems (26) and (27) are UGAS, then the cascaded system (25) is also UGAS.

Proof: Note that the Lyapunov functions $V_{1}\left(\boldsymbol{x}_{1}\right)=\boldsymbol{x}_{1}^{T} P_{1} \boldsymbol{x}_{1}$ and $V_{2}\left(\boldsymbol{x}_{2}\right)=\boldsymbol{x}_{2}^{T} P_{2} \boldsymbol{x}_{2}$ for the subsystems (26) and (27) satisfy Assumptions 1 and 4 and ensure exponential stability of the individual subsystems. Equations (14), as well as (24) and (25), are special cases of (8), where the individual subsystems 
$\boldsymbol{f}_{1}\left(\boldsymbol{x}_{1}\right)$ and $\boldsymbol{f}_{2}\left(\boldsymbol{x}_{2}\right)$ are represented by fuzzy models. The interconnection term $\boldsymbol{g}$ is a nonlinear combination of local linear models.

1) Assumption 2 is satisfied as $\forall \boldsymbol{x}_{2}:\left\|\boldsymbol{x}_{2}\right\|>\mu$

$$
\begin{aligned}
\left\|\frac{\partial V_{2}}{\partial \boldsymbol{x}_{2}}\right\|\left\|\boldsymbol{x}_{2}\right\| & =2\left\|\boldsymbol{x}_{2}^{T}\right\|\left\|P_{2}\right\|\left\|\boldsymbol{x}_{2}\right\| \\
& \leq 2 \lambda_{\max }\left(P_{2}\right)\left\|\boldsymbol{x}_{2}\right\|^{2} \leq c_{1} V_{2}\left(\boldsymbol{x}_{2}\right)
\end{aligned}
$$

for any $c_{1} \geq\left[2 \lambda_{\max }\left(P_{2}\right) / \lambda_{\min }\left(P_{2}\right)\right]$. For the second condition of Assumption 2, we have $\forall \boldsymbol{x}_{2}:\left\|\boldsymbol{x}_{2}\right\| \leq \mu$

$$
\left\|\frac{\partial V_{2}}{\partial \boldsymbol{x}_{2}}\right\|=\left\|2 \boldsymbol{x}_{2}^{T} P_{2}\right\| \leq 2\left\|\boldsymbol{x}_{2}\right\|\left\|P_{2}\right\| \leq 2 \mu \lambda_{\max }\left(P_{2}\right)=c_{2} \text {. }
$$

2) Consider continuous, positive functions $\theta_{1}\left(\left\|\boldsymbol{x}_{1}\right\|\right)=$ $\max _{\boldsymbol{z}}\left\|A_{21}(\boldsymbol{z})\right\|\left\|\boldsymbol{x}_{1}\right\|$ and $\theta_{2}\left(\left\|\boldsymbol{x}_{1}\right\|\right)=0$. By choosing these functions, we can ensure that $\|\boldsymbol{g}(\boldsymbol{x})\|=\sum_{i=1}^{m}$ $w_{i}(\boldsymbol{z}) A_{21} \boldsymbol{x}_{1} \leq \theta_{1}\left(\left\|\boldsymbol{x}_{1}\right\|\right)+\theta_{2}\left(\left\|\boldsymbol{x}_{1}\right\|\right)\left\|\boldsymbol{x}_{2}\right\|$, and therefore, Assumption 3 is satisfied.

Since the conditions of Theorem 1 are satisfied, the cascaded system is UGAS. Furthermore, since these Lyapunov functions ensure exponential stability of the subsystems, based on Proposition 1, the cascaded system is also exponentially stable.

While it is true that the cascaded system is stable under the aforementioned conditions, finding a Lyapunov function valid for the cascaded system is not trivial. A global Lyapunov function of the form

$$
V_{0}\left(\boldsymbol{x}_{1}, \boldsymbol{x}_{2}\right)=V_{1}\left(\boldsymbol{x}_{1}\right)+V_{2}\left(\boldsymbol{x}_{2}\right)+\Psi\left(\boldsymbol{x}_{1}, \boldsymbol{x}_{2}\right)
$$

has been proposed in [23], with $V_{1}$ and $V_{2}$ being Lyapunov functions for the systems (26) and (27), respectively. The cross-term $\Psi\left(\boldsymbol{x}_{1}, \boldsymbol{x}_{2}\right)$ has been constructed by the authors of [23], under the condition that the cascaded system satisfies Assumptions 2 and 3 .

For the case when the first subsystem is linear and timeinvariant, the authors of [23] proved that the cross-term exists and is continuous, and $V_{0}$ is positive definite and radially unbounded. If (26) is globally exponentially stable, the result from [23] can be extended to the system (25). The cross-term $\Psi$ is then given by

$$
\Psi\left(\boldsymbol{x}_{1}, \boldsymbol{x}_{2}\right)=\int_{0}^{\infty} \frac{\partial V_{2}}{\partial \boldsymbol{x}_{2}}\left(\tilde{\boldsymbol{x}}_{2}(s)\right) A_{21}(\boldsymbol{z}(s)) \tilde{\boldsymbol{x}}_{1}(s) d s
$$

where $\tilde{\boldsymbol{x}}_{1}$ and $\tilde{\boldsymbol{x}}_{2}$ are the trajectories of systems (26) and (27), respectively.

It has to be noted that in general, the sum of the individual Lyapunov function is not a Lyapunov function for the cascaded system. To use the sum of Lyapunov functions would require additional, unnecessary constraints.

In the same way, for Theorems 4 and 5, the stability conditions presented in Section III can be relaxed. The new conditions are presented next.

The conditions of Theorem 4 can be replaced as follows.

Theorem 10: Consider system (24) expressed as

$$
\dot{\boldsymbol{x}}=\left(\begin{array}{cc}
A_{1}\left(\boldsymbol{z}_{1}\right) & 0 \\
0 & A_{2}\left(\boldsymbol{z}_{1}, \boldsymbol{z}_{2}\right)
\end{array}\right) \boldsymbol{x}+\left(\begin{array}{c}
0 \\
A_{21}\left(\boldsymbol{z}_{1}, \boldsymbol{z}_{2}\right)
\end{array}\right) \boldsymbol{x}_{1}
$$

where $A_{1}\left(\boldsymbol{z}_{1}\right)=\sum_{i=1}^{m} w_{i}\left(\boldsymbol{z}_{1}\right) A_{1 i}, \quad A_{2}\left(\boldsymbol{z}_{1}, \boldsymbol{z}_{2}\right)=\sum_{i=1}^{m} w_{i}$ $\left(\boldsymbol{z}_{1}, \boldsymbol{z}_{2}\right) A_{2}$, etc. This system is UGAS if there exist $P_{1}=\stackrel{P_{1}^{T}>}{>}$ $0, P_{2}=P_{2}^{T}>0$ so that

$$
\begin{aligned}
& A_{1 i}^{T} P_{1}+P_{1} A_{1 i}<0 \\
& A_{2 i}^{T} P_{2}+P_{2} A_{2 i}<0
\end{aligned}
$$

for $i=1,2, \ldots, m$.

The proof is similar to that of Theorem 9 .

In order to relax the conditions of Theorem 5, let $K_{1}$ and $K_{2}$ be the number of operating and interpolation regimes for the individual subsystems, with $K_{1}^{i}$ and $K_{2}^{j}$ being the index sets corresponding to the local models of the subsystems active in the matching region. Note that, in general, the number of regions generated in such a way is lesser than the number of regions for the global system, i.e., $K_{1}+K_{2} \leq K$. Then, the conditions can be expressed as follows.

Proposition 2: The system (16) is UGAS if there exist matrices $P_{1}^{i}=\left(P_{1}^{i}\right)^{T}>0, P_{2}^{j}=\left(P_{2}^{j}\right)^{T}>0, H_{1}=H_{1}^{T}>0$, $H_{2}=H_{2}^{T}>0, F_{1}^{i}$, and $F_{2}^{j}, i=1,2, \ldots, K_{1}, j=1,2, \ldots, K_{2}$ so that

$$
\begin{aligned}
& P_{1}^{i}=\left(F_{1}^{i}\right)^{T} H_{1} F_{1}^{i} \\
& P_{2}^{j}=\left(F_{2}^{j}\right)^{T} H_{2} F_{2}^{j} \\
& F_{1}^{i} \boldsymbol{x}_{1}=F_{1}^{t} \boldsymbol{x}_{1} \quad \forall \boldsymbol{x}_{1} \in X_{1}^{i} \cap X_{1}^{t} \\
& F_{2}^{j} \boldsymbol{x}_{2}=F_{2}^{l} \boldsymbol{x}_{2} \quad \forall \boldsymbol{x}_{2} \in X_{2}^{j} \cap X_{2}^{l} \\
& A_{1 k}^{T} P_{1}^{i}+P_{1}^{i} A_{1 k}<0 \quad \forall k \in K_{1}^{i} \\
& A_{2 k}^{T} P_{2}^{j}+P_{2}^{j} A_{2 k}<0 \quad \forall k \in K_{2}^{j} .
\end{aligned}
$$

Note that the proposed conditions are still only sufficient conditions for the stability of cascaded fuzzy systems. However, by taking advantage of the special form of the system, i.e., studying the subsystems instead of the overall fuzzy system, the complexity of the associated LMI problem is reduced with respect to Theorems 2, 4, and 5 .

In the remainder of this section, we study the convergence rate of the system (25) with respect to the convergence rate of the individual subsystems (26) and (27).

Consider the case when both subsystems are exponentially stable, i.e., there exist $\beta_{1}, \beta_{2}, \gamma_{1}, \gamma_{2}>0$ so that

$$
\begin{aligned}
\left\|\boldsymbol{x}_{1}\right\| & \leq \beta_{1}\left\|\boldsymbol{x}_{10}\right\| e^{-\gamma_{1} t} \\
\left\|\boldsymbol{x}_{2}\right\| & \leq \beta_{2}\left\|\boldsymbol{x}_{20}\right\| e^{-\gamma_{2} t} .
\end{aligned}
$$

It has been an open question whether this also meant that the convergence rate of the system (25) is $\min \left\{\gamma_{1}, \gamma_{2}\right\}$; this conjecture is indeed valid for linear systems; however, it cannot be proven with a Lyapunov function of the form $V(\boldsymbol{x})=$ $V_{1}\left(\boldsymbol{x}_{1}\right)+V_{2}\left(\boldsymbol{x}_{2}\right)$, where $V_{1}$ and $V_{2}$ are Lyapunov functions for the individual subsystems, as presented next.

Consider the joint system (25), and assume that there exist a Lyapunov function, $V=\boldsymbol{x}^{T} P \boldsymbol{x}, P=P^{T}>0$, and $\gamma>0$ so that

$$
\begin{aligned}
& \alpha\|\boldsymbol{x}\|^{2} \leq V \leq \beta\|\boldsymbol{x}\|^{2} \\
& \dot{V}=\boldsymbol{x}^{T}\left(A(\boldsymbol{z})^{T} P+A(\boldsymbol{z})\right) \boldsymbol{x}=-\boldsymbol{x}^{T} Q(\boldsymbol{z}) \boldsymbol{x} \leq-\gamma\|\boldsymbol{x}\|^{2} .
\end{aligned}
$$


Then, the convergence rate of the system is at least $\gamma / \beta$. To have the same convergence rate for the subsystems, it is necessary (in terms of the earlier conditions) that there exist $P_{1}=P_{1}^{T}>0$, $P_{2}=P_{2}^{T}>0$ so that

1) $\alpha \leq \lambda_{\min }\left(P_{1}, P_{2}\right)$;

2) $\beta \geq \lambda_{\max }\left(P_{1}, P_{2}\right)$

3) $\lambda_{\max }\left(\operatorname{diag}\left[A_{1}\left(\boldsymbol{z}_{1}\right)^{T} P_{1}+P_{1} A_{1}\left(\boldsymbol{z}_{1}\right), A_{2}\left(\boldsymbol{z}_{2}\right)^{T} P_{2}+P_{2} A_{2}\right.\right.$ $\left.\left(\boldsymbol{z}_{2}\right)\right] \leq-\gamma$.

Now, it will be proven that, if the subsystems are exponentially stable, the convergence rate of the system (24) is also determined by the convergence rate of the individual subsystems (26) and (27).

Theorem 11: The convergence rate of the system (25) is equal to $\max \left\{-\alpha_{1},-\alpha_{2}\right\}$, if

1) system (26) is exponentially stable with convergence rate $-\alpha_{1}$

2) system (27) is exponentially stable with convergence rate $-\alpha_{2}$;

3) the matrix $A_{21}(\boldsymbol{z})$ is bounded, i.e., there exists $M \in \mathbb{R}$, so that $\forall \boldsymbol{z},\left\|A_{21}(\boldsymbol{z})\right\| \leq M$.

Proof: Assumption 1 can be written as $\left\|\boldsymbol{x}_{1}(t)\right\| \leq$ $k_{1}\left\|\boldsymbol{x}_{10}\right\| e^{-\alpha_{1} t}$. The solution of the system (27) is the homogeneous solution $\boldsymbol{x}_{2 \mathrm{~h}}(t)$ of the system

$$
\dot{\boldsymbol{x}}_{2}=A_{21}(\boldsymbol{z}) \boldsymbol{x}_{1}+A_{2}(\boldsymbol{z}) \boldsymbol{x}_{2}
$$

and therefore, it satisfies $\left\|\boldsymbol{x}_{2 \mathrm{~h}}(t)\right\| \leq k_{2}\left\|\boldsymbol{x}_{20}\right\| e^{-\alpha_{2} t}$. The particular solution of (32) can be expressed as

$$
\boldsymbol{x}_{2 \mathrm{p}}=\int_{t_{0}}^{t} \boldsymbol{x}_{2 \mathrm{~h}}(t-s) A_{21}(\boldsymbol{z}(s)) \boldsymbol{x}_{1}(s) d s .
$$

Then

$$
\begin{aligned}
\left\|\boldsymbol{x}_{2 \mathrm{p}}\right\| & =\left\|\int_{t_{0}}^{t} \boldsymbol{x}_{2 \mathrm{~h}}(t-s) A_{21}(\boldsymbol{z}(s)) \boldsymbol{x}_{1}(s) d s\right\| \\
& \leq \int_{t_{0}}^{t}\left\|\boldsymbol{x}_{2 \mathrm{~h}}(t-s)\right\|\left\|A_{21}(\boldsymbol{z}(s))\right\|\left\|\boldsymbol{x}_{1}(s)\right\| d s \\
& \leq \int_{t_{0}}^{t} k_{2}\left\|\boldsymbol{x}_{20}\right\| e^{-\alpha_{2}(t-s)} M k_{1}\left\|\boldsymbol{x}_{10}\right\| e^{-\alpha_{1} s} d s \\
& =k_{1} k_{2} M\left\|\boldsymbol{x}_{10}\right\|\left\|\boldsymbol{x}_{20}\right\| e^{-\alpha_{2} t} \int_{t_{0}}^{t} e^{\left(\alpha_{2}-\alpha_{1}\right) s} d s .
\end{aligned}
$$

If $\alpha_{2} \neq \alpha_{1}$

$$
\begin{aligned}
&\left\|\boldsymbol{x}_{2 \mathrm{p}}\right\|= k_{1} k_{2} M\left\|\boldsymbol{x}_{10}\right\|\left\|\boldsymbol{x}_{20}\right\|\left|\left(\alpha_{2}-\alpha_{1}\right)\right|^{-1} \\
& \cdot e^{-\alpha_{2} t}\left(e^{\left(\alpha_{2}-\alpha_{1}\right) t}-e^{\left(\alpha_{2}-\alpha_{1}\right) t_{0}}\right) \\
&=k_{1} k_{2} M\left\|\boldsymbol{x}_{10}\right\|\left\|\boldsymbol{x}_{20}\right\|\left|\left(\alpha_{2}-\alpha_{1}\right)\right|^{-1}\left|\left(e^{-\alpha_{1} t}-c_{1} e^{-\alpha_{2} t}\right)\right|
\end{aligned}
$$

where $c_{1}=e^{\left(\alpha_{2}-\alpha_{1}\right) t_{0}}$.

A bound on the general solution of (32) is

$$
\begin{aligned}
\left\|\boldsymbol{x}_{2}\right\| \leq & \left\|\boldsymbol{x}_{2 \mathrm{~h}}\right\|+\left\|\boldsymbol{x}_{2 \mathrm{p}}\right\| \\
\leq & k_{2}\left\|\boldsymbol{x}_{20}\right\| e^{-\alpha_{2} t}+k_{1} k_{2} M\left\|\boldsymbol{x}_{10}\right\|\left\|\boldsymbol{x}_{20}\right\| \\
& \quad \cdot\left|\left(\alpha_{2}-\alpha_{1}\right)\right|^{-1}\left|\left(e^{-\alpha_{1} t}-c_{1} e^{-\alpha_{2} t}\right)\right| \\
& \leq c_{2} e^{\max \left\{-\alpha_{1},-\alpha_{2}\right\} t}
\end{aligned}
$$

where $c_{2}=\max \left\{k_{2}\left\|\boldsymbol{x}_{20}\right\|\left(1+k_{1} M\left\|\boldsymbol{x}_{10}\right\|\left|\left(\alpha_{2}-\alpha_{1}\right)\right|^{-1} c_{1}\right)\right.$, $\left.k_{1} k_{2}\left\|\boldsymbol{x}_{10}\right\|\left\|\boldsymbol{x}_{20}\right\| M\left|\left(\alpha_{2}-\alpha_{1}\right)\right|^{-1}\right\}$.

For $\alpha_{1}=\alpha_{2}=\alpha$, we have

$$
\begin{aligned}
& \left\|\boldsymbol{x}_{2 \mathrm{p}}\right\| \leq k_{1} k_{2} M\left\|\boldsymbol{x}_{10}\right\|\left\|\boldsymbol{x}_{20}\right\| e^{-\alpha t}\left(t-t_{0}\right) \\
& \left\|\boldsymbol{x}_{2}\right\| \leq\left\|\boldsymbol{x}_{2 \mathrm{~h}}\right\|+\left\|\boldsymbol{x}_{2 \mathrm{p}}\right\| \\
& \leq k_{2}\left\|\boldsymbol{x}_{20}\right\| e^{-\alpha t}+k_{1} k_{2} M\left\|\boldsymbol{x}_{10}\right\|\left\|\boldsymbol{x}_{20}\right\| e^{-\alpha t}\left(t-t_{0}\right) \\
& \leq c_{3} e^{-\alpha t}+c_{4} t e^{-\alpha t}
\end{aligned}
$$

with $c_{3}=k_{2}\left\|\boldsymbol{x}_{20}\right\|$ and $c_{4}=k_{1} k_{2}\left\|\boldsymbol{x}_{10}\right\|\left\|\boldsymbol{x}_{20}\right\| M$. For the bound in (33), it has been shown that the convergence rate is $\alpha$ [32].

This means that the convergence rate of the system (32), and therefore, of the system (25) is determined by the convergence rate of the individual subsystems.

\section{B. Cascaded Observers}

This section presents the cascaded approach applied to observer design for TS fuzzy systems. As before, consider the fuzzy system with normal membership functions

$$
\begin{aligned}
& \dot{\boldsymbol{x}}=\sum_{i=1}^{m} w_{i}(\boldsymbol{z})\left(A_{i} \boldsymbol{x}+B_{i} \boldsymbol{u}+a_{i}\right) \\
& \boldsymbol{y}=\sum_{i=1}^{m} w_{i}(\boldsymbol{z})\left(C_{i} \boldsymbol{x}+d_{i}\right)
\end{aligned}
$$

and a fuzzy observer of the form

$$
\begin{aligned}
& \dot{\widehat{\boldsymbol{x}}}=\sum_{i=1}^{m} w_{i}(\boldsymbol{z})\left(A_{i} \widehat{\boldsymbol{x}}+B_{i} \boldsymbol{u}+a_{i}+L_{i}(\boldsymbol{y}-\widehat{\boldsymbol{y}})\right) \\
& \widehat{\boldsymbol{y}}=\sum_{i=1}^{m} w_{i}(\boldsymbol{z})\left(C_{i} \widehat{\boldsymbol{x}}+d_{i}\right) .
\end{aligned}
$$

Assuming that the system matrices for each rule $i=1,2, \ldots, m$ can be written as

$$
\begin{aligned}
A_{i} & =\left(\begin{array}{cc}
A_{1} & 0 \\
A_{21} & A_{2}
\end{array}\right)_{i}=\left(\begin{array}{cc}
A_{1 i} & 0 \\
A_{21_{i}} & A_{2 i}
\end{array}\right) \\
C_{i} & =\left(\begin{array}{cc}
C_{1} & 0 \\
C_{21} & C_{2}
\end{array}\right)_{i}=\left(\begin{array}{cc}
C_{1 i} & 0 \\
C_{21_{i}} & C_{2_{i}}
\end{array}\right)
\end{aligned}
$$

observers can be designed individually for each subsystem and each rule, with the overall observer gain having the form

$$
L_{i}=\left(\begin{array}{cc}
L_{1} & 0 \\
0 & L_{2}
\end{array}\right)_{i}=\left(\begin{array}{cc}
L_{1 i} & 0 \\
0 & L_{2 i}
\end{array}\right)
$$

where $i$ denotes the rule number.

Again, two cases are distinguished. If the weights do not depend on the states to be estimated, the cascaded error system can be written as

$$
\begin{aligned}
\dot{\boldsymbol{e}} & =\sum_{i=1}^{m} \sum_{j=1}^{m} w_{i}(\boldsymbol{z}) w_{j}(\boldsymbol{z})\left(A_{i}-L_{i} C_{j}\right) \boldsymbol{e} \\
& =\sum_{i=1}^{m} \sum_{j=1}^{m} w_{i}(\boldsymbol{z}) w_{j}(\boldsymbol{z})\left(\begin{array}{cc}
A_{1 i}-L_{1 i} C_{1 j} & 0 \\
A_{21 i}-L_{2 i} C_{21_{j}} & A_{2 i}-L_{2 i} C_{2_{j}}
\end{array}\right) \boldsymbol{e} .
\end{aligned}
$$


This system is of the form (24) for which the stability conditions from Section IV-A can be used. If the $C$ matrix is common for all the rules, the presented theorems can be directly applied.

In the case when the scheduling vector does not depend on the states to be estimated, Theorem 11 can also be applied to the design of observers with guaranteed convergence rate.

Using the results on the convergence rate, Theorem 7 can be reformulated as follows.

Theorem 12: The decay rate of the error system (36) is at least $\alpha$ if there exist $P_{1}=P_{1}^{T}>0$ and $P_{2}=P_{2}^{T}>0$ so that for $i=1,2, \ldots, m$

$$
\begin{aligned}
& \left(A_{1 i}-L_{1 i} C_{1 i}\right)^{T} P_{1}+P_{1}\left(A_{1 i}-L_{1 i} C_{1 i}\right)+2 \alpha P_{1}<0 \\
& \left(A_{2 i}-L_{2 i} C_{2 i}\right)^{T} P_{2}+P_{2}\left(A_{2 i}-L_{2 i} C_{2 i}\right)+2 \alpha P_{2}<0 \\
& \left(A_{1 i}-L_{1 i} C_{1 j}\right)^{T} P_{1}+P_{1}\left(A_{1 i}-L_{1 i} C_{1 j}\right)+2 \alpha P_{1}<0 \\
& j=1,2, \ldots, m, \quad w_{1 i}\left(\boldsymbol{z}_{1}\right) w_{1 j}\left(\boldsymbol{z}_{1}\right) \neq 0 \\
& \left(A_{2 i}-L_{2 i} C_{2 j}\right)^{T} P_{2}+P_{2}\left(A_{2 i}-L_{2 i} C_{2 j}\right)+2 \alpha P_{2}<0 \\
& j=1,2, \ldots, m, \quad w_{2 i}(\boldsymbol{z}) w_{2 j}(\boldsymbol{z}) \neq 0 .
\end{aligned}
$$

The proof follows directly. The previous conditions explicitly state that, in order to design a global observer with a desired convergence rate, it is sufficient to design observers for the subsystems with the same convergence rate.

Now, consider the case when the parameters $z$ depend on the states to be estimated, i.e., $\boldsymbol{z}=\widehat{z}$. For simplicity, only the case with common measurement matrix is considered. Then, the fuzzy system is expressed as

$$
\begin{aligned}
\dot{\boldsymbol{x}} & =\sum_{i=1}^{m} w_{i}(\boldsymbol{z})\left(A_{i} \boldsymbol{x}+B_{i} \boldsymbol{u}+a_{i}\right) \\
\boldsymbol{y} & =C \boldsymbol{x}
\end{aligned}
$$

and the error system can be written as

$$
\begin{aligned}
\dot{\boldsymbol{e}}= & \sum_{i=1}^{m}\left(\begin{array}{cc}
w\left(\widehat{\boldsymbol{z}}_{1}\right)\left(A_{1}-L_{1} C_{1}\right) & 0 \\
w(\widehat{\boldsymbol{z}})\left(A_{21}-L_{2} C_{21}\right) & w(\widehat{\boldsymbol{z}})\left(A_{2}-L_{2} C_{2}\right)
\end{array}\right)_{i} \boldsymbol{e} \\
& +\sum_{i=1}^{m}\left(\begin{array}{c}
w_{1 i}\left(\boldsymbol{z}_{1}\right)-w_{1 i}\left(\widehat{\boldsymbol{z}}_{1}\right) \\
w_{2 i}(\boldsymbol{z})-w_{2 i}(\widehat{\boldsymbol{z}})
\end{array}\right)\left(A_{i} \boldsymbol{x}+B_{i} \boldsymbol{u}+a_{i}\right) .
\end{aligned}
$$

To ensure the stability of the observer in such a case, Theorem 8 can be applied. However, using the results for cascaded systems, relaxed stability conditions are derived. These conditions can be expressed as follows.

Theorem 13: The cascaded error system (38) is UGAS if there exist a Lyapunov function $V_{1}\left(\boldsymbol{x}_{1}\right), P_{2}=P_{2}^{T}>0$ and two continuous functions $\theta_{1}, \theta_{2}: \mathcal{R}^{+} \rightarrow \mathcal{R}^{+}$such that the following hold.

1) The Lyapunov function $V_{1}$ ensures exponential stability of the error system

$$
\begin{aligned}
\dot{\boldsymbol{e}}_{1}= & \sum_{i=1}^{m} w_{1 i}\left(\widehat{\boldsymbol{z}}_{1}\right)\left(A_{1}-L_{1} C_{1}\right) \boldsymbol{e}_{1}+\left(w_{1 i}\left(\boldsymbol{z}_{1}\right)\right. \\
& \left.-w_{1 i}\left(\widehat{\boldsymbol{z}}_{1}\right)\right)\left(A_{1 i} \boldsymbol{x}_{1}+B_{1_{i}} \boldsymbol{u}+a_{1 i}\right) .
\end{aligned}
$$

2) $P_{2}$ satisfies $\left(A_{2 i}\right)^{T} P_{2}+P_{2} A_{2 i}<0, i=1,2, \ldots, m$.
3) $\| \sum_{i=1}^{m}\left(w_{2 i}\left(\boldsymbol{z}_{1}, \boldsymbol{z}_{2}\right)-w_{2 i}\left(\widehat{\boldsymbol{z}}_{1}, \widehat{\boldsymbol{z}}_{2}\right)\right)\left(A_{21 i} \boldsymbol{x}_{1}+A_{2 i} \boldsymbol{x}_{2}+\right.$ $\left.B_{2 i} \boldsymbol{u}+a_{2 i}\right)\left\|\leq \theta_{1}\left(\left\|\boldsymbol{e}_{1}\right\|\right)+\theta_{2}\left(\left\|\boldsymbol{e}_{1}\right\|\right)\right\| \boldsymbol{e}_{2} \|$.

Proof: Since $\left(A_{2 i}\right)^{T} P_{2}+P_{2} A_{2 i}<0, i=1,2, \ldots, m, V_{2}$ is a Lyapunov function for

$$
\dot{\boldsymbol{e}}_{2}=\sum_{i=1}^{m} w_{2 i}\left(\widehat{\boldsymbol{z}}_{1}, \widehat{\boldsymbol{z}}_{2}\right)\left(A_{2}-L_{2} C_{2}\right) \boldsymbol{e}_{2}
$$

and this system is UGAS (see Assumption 1). Let $c_{1}=$ $2\left[\lambda_{\max }\left(P_{2}\right) / \lambda_{\min }\left(P_{2}\right)\right]$, where $\lambda_{\max }$ is the eigenvalue with the largest absolute value and $c_{2}=2 \eta \lambda_{\max }\left(P_{2}\right)$. With these constants, Assumption 2 is satisfied. The Lyapunov function $V_{1}$ satisfies Assumption 4.

Now, the interconnection term in the second subsystem can be written as

$$
\begin{aligned}
g\left(\boldsymbol{e}_{1}, \boldsymbol{e}_{2}\right)= & \sum_{i=1}^{m} w_{2 i}\left(\widehat{\boldsymbol{z}}_{1}, \widehat{\boldsymbol{z}}_{2}\right)\left(A_{21 i}-L_{2 i} C_{2 i}\right) \boldsymbol{e}_{1} \\
& +\sum_{i=1}^{m}\left(w_{2 i}\left(\boldsymbol{z}_{1}, \boldsymbol{z}_{2}\right)-w_{2 i}\left(\widehat{\boldsymbol{z}}_{1}, \widehat{\boldsymbol{z}}_{2}\right)\right) \\
& \left(A_{21 i} \boldsymbol{x}_{1}+A_{2 i} \boldsymbol{x}_{2}+B_{2 i} \boldsymbol{u}+a_{2 i}\right) \\
\left\|g\left(\boldsymbol{e}_{1}, \boldsymbol{e}_{2}\right)\right\| \leq & \sum_{i=1}^{m}\left\|w_{2 i}\left(\widehat{\boldsymbol{z}}_{1}, \widehat{\boldsymbol{z}}_{2}\right)\right\|\left\|A_{21 i}-L_{2 i} C_{2 i}\right\|\left\|\boldsymbol{e}_{1}\right\| \\
& +\theta_{1}\left(\left\|\boldsymbol{e}_{1}\right\|\right)+\theta_{2}\left(\left\|\boldsymbol{e}_{1}\right\|\right)\left\|\boldsymbol{e}_{2}\right\| \\
\left\|g\left(\boldsymbol{e}_{1}, \boldsymbol{e}_{2}\right)\right\| \leq & \tau\left\|\boldsymbol{e}_{1}\right\|+\theta_{1}\left(\left\|\boldsymbol{e}_{1}\right\|\right)+\theta_{2}\left(\left\|\boldsymbol{e}_{1}\right\|\right)\left\|\boldsymbol{e}_{2}\right\| \\
\left\|g\left(\boldsymbol{e}_{1}, \boldsymbol{e}_{2}\right)\right\| \leq & \theta_{1}^{\prime}\left(\left\|\boldsymbol{e}_{1}\right\|\right)+\theta_{2}\left(\left\|\boldsymbol{e}_{1}\right\|\right)\left\|\boldsymbol{e}_{2}\right\| .
\end{aligned}
$$

With this, Assumption 3 [see (10)] is satisfied, and based on Theorem 1, the cascaded system is UGAS. Moreover, since the first subsystem is exponentially stable, the cascaded system is also exponentially stable (see Proposition 1).

\section{Design Using LMI Regions}

Designing observers based on the conditions presented in the previous sections may not give an acceptable performance, since the poles of the observer may be placed at arbitrary locations in the left half-plane. This problem can be avoided by using LMI regions, i.e., constraining the poles of each local model to a specific region. A definition of the LMI regions can be found in [33].

Definition 3: A subset $\mathcal{D}$ of the complex plane is called an LMI region if there exist a symmetric matrix $\alpha \in \mathcal{R}^{m \times m}$ and a matrix $\beta \in \mathcal{R}^{m \times m}$ such that

$$
\mathcal{D}=\left\{z \in \mathcal{C}: f_{\mathcal{D}}(z)<0\right\}
$$

where

$$
f_{\mathcal{D}}(z)=\alpha+z \beta+\bar{z} \beta^{T}
$$

is the characteristic function of the LMI region.

One can easily see that, because of the form of the function $f_{\mathcal{D}}(z)$, LMI regions are convex and symmetric with respect to the real axis. Useful LMI regions include a vertical strip $\left[d_{\mathrm{l}}, d_{\mathrm{u}}\right]$ and a conic sector centered in the origin with inner angle $\theta$ 


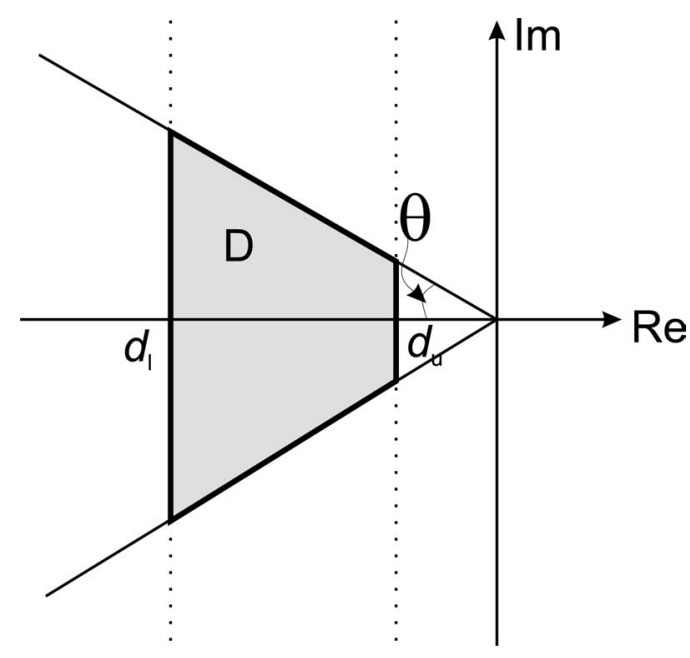

Fig. 3. LMI regions.

(see Fig. 3). If all the eigenvalues of a matrix $A$ are located in a region $\mathcal{D}$, then the matrix $A$ is called $\mathcal{D}$-stable.

A theorem to ensure $\mathcal{D}$-stability of a matrix $A$ was given in [33].

Theorem 14: The matrix $A$ is $\mathcal{D}$-stable if and only if there exists $P=P^{T}>0$ so that

$$
\alpha \otimes P+\beta \otimes A P+\beta^{T} \otimes(A P)^{T}<0
$$

where $\otimes$ is the Kronecker product.

In the context of observer design, using LMI regions to ensure the specific $\mathcal{D}$-stability of the observer effectively means adding constraints to the presented LMI problems; more specifically

$$
\begin{aligned}
& {\left[\alpha_{j, k} P+\beta_{j, k} P\left(A_{i}-L_{i} C_{i}\right)+\beta_{k, j}\left(A_{i}-L_{i} C_{i}\right)^{T} P\right]<0} \\
& j, k=1,2, \ldots, m .
\end{aligned}
$$

Here, $\alpha_{j, k}$ and $\beta_{j, k}$ denote the $(j, k)$ th element of the corresponding matrices.

\section{EXAMPLES}

In this section, we demonstrate the benefits of the proposed approach on two simulated examples.

\section{A. Feasibility}

We illustrate on a numerical example the case when the proposed conditions can be used to prove the stability of a fuzzy system, which otherwise requires much more complicated analysis.

Consider the fuzzy system

$$
\dot{\boldsymbol{x}}=\sum_{i=1}^{2} w_{i}(\boldsymbol{z}) A_{i} \boldsymbol{x}
$$

with $w_{1}(\boldsymbol{z}) \geq 0, w_{2}(\boldsymbol{z}) \geq 0, w_{1}(\boldsymbol{z})+w_{2}(\boldsymbol{z})=1 \forall \boldsymbol{z}$.
The state matrices of the local linear models are given as

$$
A_{1}=\left(\begin{array}{ccccc}
-0.7 & -1.0 & 0 & 0 & 0 \\
-1.0 & -2.8 & 0 & 0 & 0 \\
-0.1 & -1.8 & -1.4 & 0.6 & 0.0 \\
0.1 & -0.7 & 0.6 & -3.1 & 0.4 \\
-1.8 & 1.3 & 0.0 & 0.4 & -1.9
\end{array}\right)
$$

and

$$
A_{2}=\left(\begin{array}{ccccc}
-3.3 & -1.3 & 0 & 0 & 0 \\
-1.3 & -2.6 & 0 & 0 & 0 \\
0 & 0 & -1.1 & 0.6 & -0.7 \\
0 & 0 & 0.6 & -5.2 & 1.7 \\
0 & 0 & -0.7 & 1.7 & -2.0
\end{array}\right) .
$$

The LMI problem

$$
\begin{aligned}
P & >0 \\
A_{1}^{T} P+P A_{1} & <0 \\
A_{2}^{T} P+P A_{2} & <0
\end{aligned}
$$

is infeasible, and therefore, Theorems 2 and 4 cannot be applied. The stability of this system can be investigated using Theorem 5.

By examining the form of the system matrices, one can easily see that the system can be cascaded, with $\boldsymbol{x}_{1}=\left[\begin{array}{ll}x_{1} & x_{2}\end{array}\right]^{T}$ and $\boldsymbol{x}_{2}=\left[\begin{array}{lll}x_{3} & x_{4} & x_{5}\end{array}\right]^{T}$.

Based on Theorem 9, the system (41) is stable if the individual subsystems are stable. As such, in order to prove the stability of the system (41), it is sufficient that the LMI problems

$$
\begin{aligned}
P_{1} & >0 \\
A_{11}^{T} P_{1}+P_{1} A_{11} & <0 \\
A_{12}^{T} P_{1}+P_{1} A_{12} & <0
\end{aligned}
$$

and

$$
\begin{aligned}
P_{2} & >0 \\
A_{12}^{T} P_{2}+P_{2} A_{12} & <0 \\
A_{22}^{T} P_{2}+P_{2} A_{22} & <0
\end{aligned}
$$

are feasible. Using Yalmip's solvesdp [34], one can easily see that this is indeed so.

This example illustrates the main benefit of the proposed stability conditions: While the conditions imposed by conventional methods lead to an infeasible LMI system, it is still possible to prove stability of the system under study by using the stability conditions proposed in this paper.

\section{B. Observer Design}

This example of a real-world system [35] illustrates the benefits of using the cascaded approach instead of centralized observer design.

Consider the three tanks connected in a cascade, as shown in Fig. 4. Water is pumped from a reservoir into the upper tank (3). 


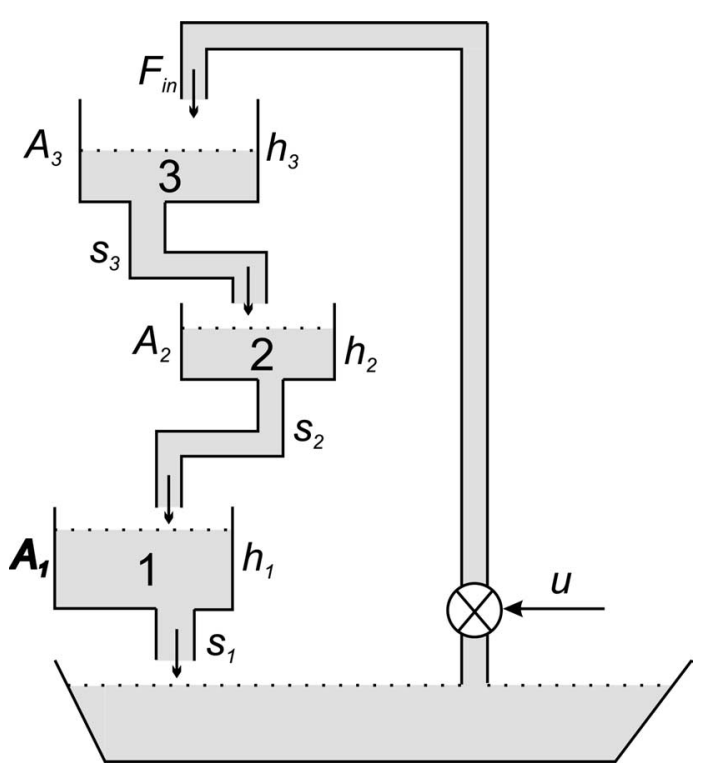

Fig. 4. Cascaded tanks system.

TABLE I

PARAMETER VALUes USED

\begin{tabular}{cccc} 
Parameter & Symbol & Value & Units \\
\hline Acceleration due to gravity & $g$ & 9.81 & $\mathrm{~m} / \mathrm{s}^{2}$ \\
Cross-sectional area tank 1 & $A_{1}$ & 10 & $\mathrm{~m}^{2}$ \\
Cross-sectional area tank 2 & $A_{2}$ & 8 & $\mathrm{~m}^{2}$ \\
Cross-sectional area tank 3 & $A_{3}$ & 9 & $\mathrm{~m}^{2}$ \\
Outlet area of tank 1 & $s_{1}$ & 0.25 & $\mathrm{~m}^{2}$ \\
Outlet area of tank 2 & $s_{2}$ & 0.2 & $\mathrm{~m}^{2}$ \\
Outlet area of tank 3 & $s_{3}$ & 0.3 & $\mathrm{~m}^{2}$ \\
Input to flow gain & $Q_{s}$ & 0.336 & $\mathrm{~m}^{3} / \mathrm{s} / \mathrm{V}$ \\
Motor time constant & $\tau$ & 3 & $\mathrm{~s}$ \\
\hline
\end{tabular}

From this tank, the water flows to the lower tanks and from the lowest tank back to the reservoir. The system has one control input $u$, which is the voltage applied to the motor of the pump and two measured outputs: the water levels $h_{3}$ in the upper tank and $h_{1}$ in the lowest tank. The flow rate $F_{\text {in }}$, provided by the pump, and the water level $h_{2}$ in the middle tank need to be estimated, and therefore, an observer has to be designed. The differential equations describing the dynamics of this system are the following:

$$
\begin{aligned}
\tau \dot{F}_{\text {in }} & =-F_{\text {in }}+Q_{s} \cdot u \\
\dot{h}_{3} & =\frac{F_{\text {in }}}{A_{3}}-\frac{s_{3} \sqrt{2 g h_{3}}}{A_{3}} \\
\dot{h}_{2} & =\frac{s_{3} \sqrt{2 g h_{3}}}{A_{2}}-\frac{s_{2} \sqrt{2 g h_{2}}}{A_{2}} \\
\dot{h}_{1} & =\frac{s_{2} \sqrt{2 g h_{2}}}{A_{1}}-\frac{s_{1} \sqrt{2 g h_{1}}}{A_{1}} .
\end{aligned}
$$

The parameter values are presented in Table I.

It is assumed that the tanks have the same height, $h_{\max }=2 \mathrm{~m}$. Therefore, all levels are bounded, $h_{i} \in\left[0, h_{\max }\right]$.

This system is highly nonlinear and a linear observer cannot be used. However, it is possible to design a fuzzy observer for this system. In order to use the proposed design, a TS fuzzy

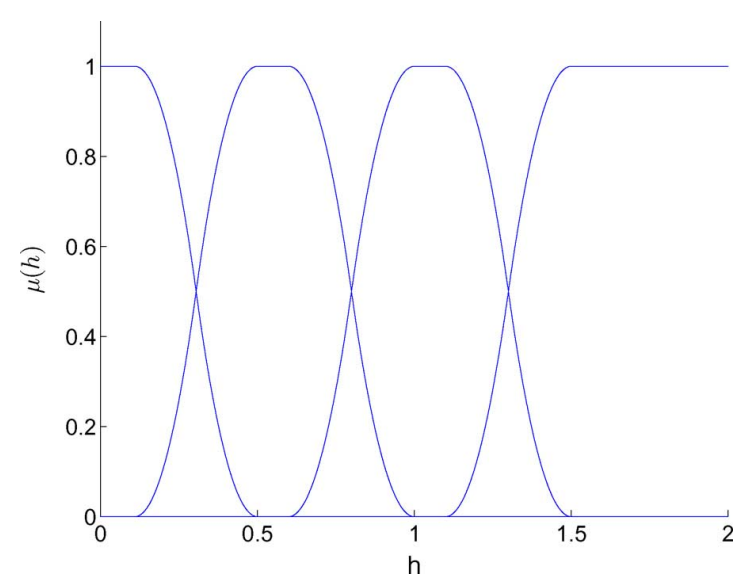

Fig. 5. Membership functions for the heights.

TABLE II

LMI REGIONS AND THE CPU TIME

\begin{tabular}{ccccrr} 
Case & $d_{l}$ & $d_{u}$ & $\theta$ & Centralized [s] & Cascaded [s] \\
\hline 1 & - & - & - & 2.73 & 0.17 \\
2 & -10 & -2 & - & 103.00 & 0.23 \\
3 & - & - & $\pi / 4$ & 1.59 & 0.22 \\
4 & -10 & -2 & $\pi / 4$ & 30.05 & 0.53 \\
5 & - & - & $\pi / 36$ & 1.75 & 0.31 \\
6 & -10 & -2 & $\pi / 36$ & 33.89 & 0.53 \\
\hline
\end{tabular}

model of the system (42) is constructed. For each level $h_{i}$, four points $h_{i} \in\{0.1,0.55,1.05,1.6\}$ are chosen, together with appropriate membership functions, as depicted in Fig. 5. Note that the scheduling vector consists of the levels $h_{1}, h_{2}$, and $h_{3}$, which are the states to be estimated.

The system (42) is linearized for each combination of the chosen points. Since the linearization is not done in equilibria, the consequents are affine. For instance, the rule obtained by linearizing in $h_{1}=0.55, h_{2}=0.1$, and $h_{3}=0.55$ is as follows.

If $h_{1}$ is approximately 0.55 and $h_{2}$ is approximately 0.1 and $h_{3}$ is approximately 0.55 , then $\dot{\boldsymbol{x}}=A \boldsymbol{x}+B u+a$, with

$$
\begin{aligned}
A & =\left(\begin{array}{cccc}
-0.3333 & 0 & 0 & 0 \\
0.1111 & -0.0995 & 0 & 0 \\
0 & 0.1120 & -0.1751 & 0 \\
0 & 0 & 0.1401 & -0.0747
\end{array}\right) \\
B & =\left(\begin{array}{llll}
0.1120 & 0 & 0 & 0
\end{array}\right)^{T} \\
a & =\left(\begin{array}{llll}
0 & -0.0547 & 0.0441 & -0.0271
\end{array}\right)^{T}
\end{aligned}
$$

where $\boldsymbol{x}=\left[\begin{array}{llll}F_{\text {in }} & h_{3} & h_{2} & h_{1}\end{array}\right]^{T}$. To compute the membership degree of the scheduling vector, the algebraic product operator is used. Note that, by using this operator, the membership functions obtained for the scheduling vectors are smooth. Other operators, such as MIN and MAX, would render the membership functions nonsmooth.

By examining the form of system (42) and the matrices of the fuzzy system, one can easily see that the system can be cascaded, with $\boldsymbol{x}_{1}=\left[\begin{array}{ll}F_{\text {in }} & h_{3}\end{array}\right]^{T}$ and $\boldsymbol{x}_{2}=\left[\begin{array}{ll}h_{2} & h_{1}\end{array}\right]^{T}$. Therefore, observers can be designed separately for the individual subsystems. The observers are designed both for the whole system and the individual subsystems using the same pole-placement method and conditions. Both observers have the form (22). 


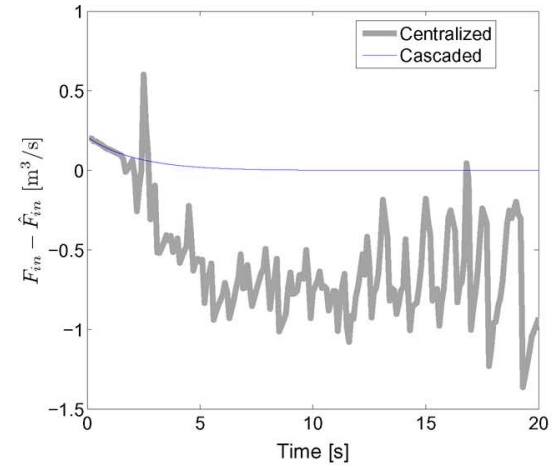

(a)

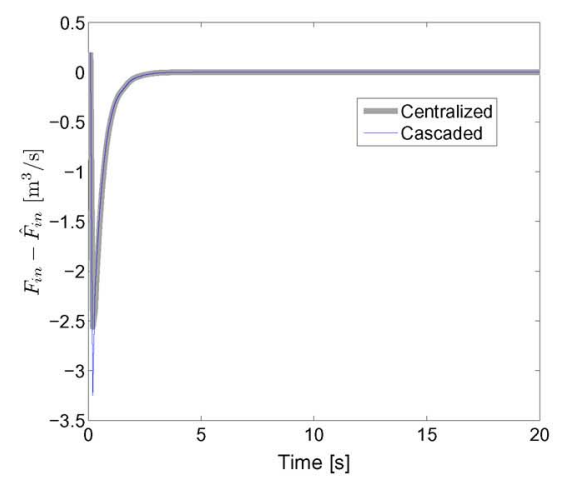

(d)

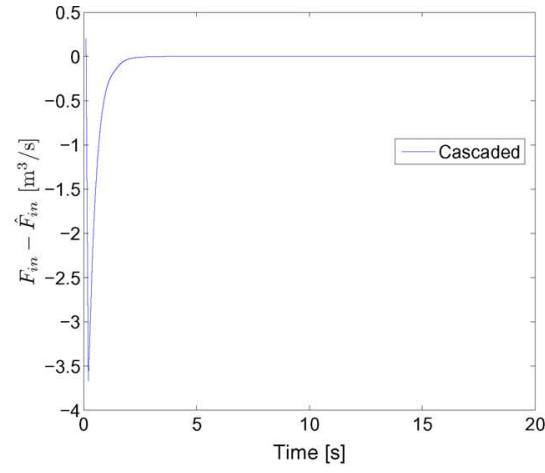

(b)

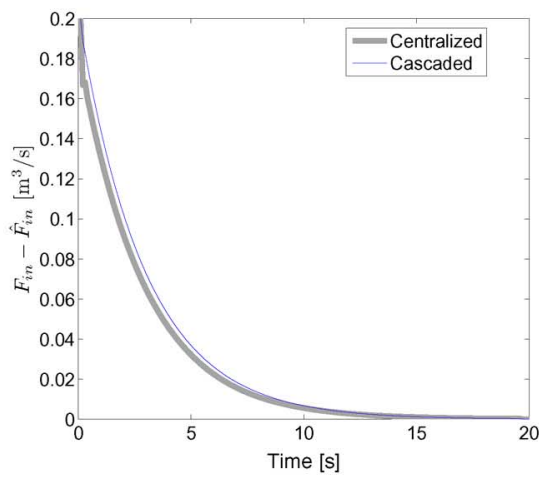

(e)

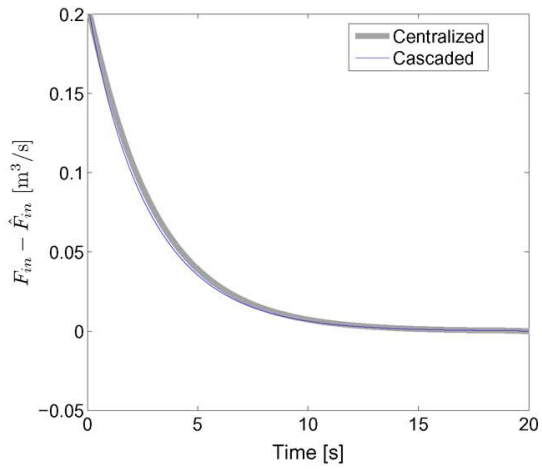

(c)

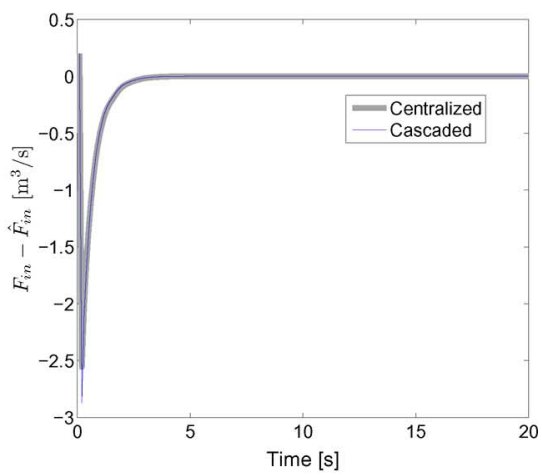

(f)

Fig. 6. Estimation errors for $F_{\text {in }}$ using centralized and cascaded observers. (a) Case 1. (b) Case 2. (c) Case 3. (d) Case 4. (e) Case 5. (f) Case 6.

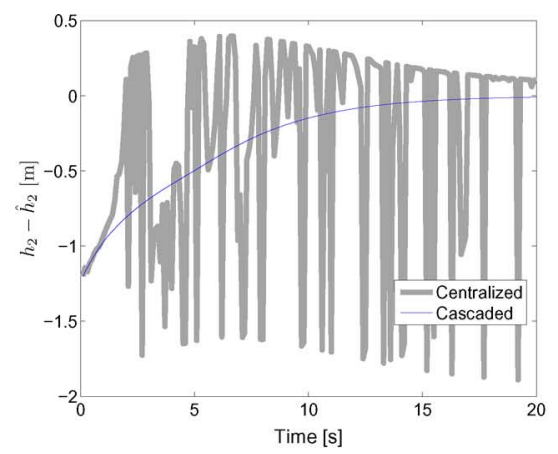

(a)

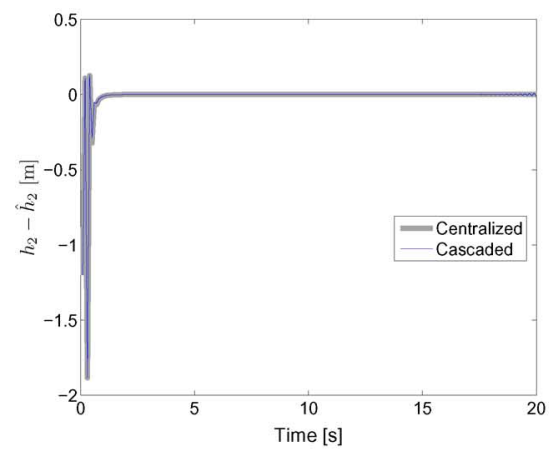

(d)

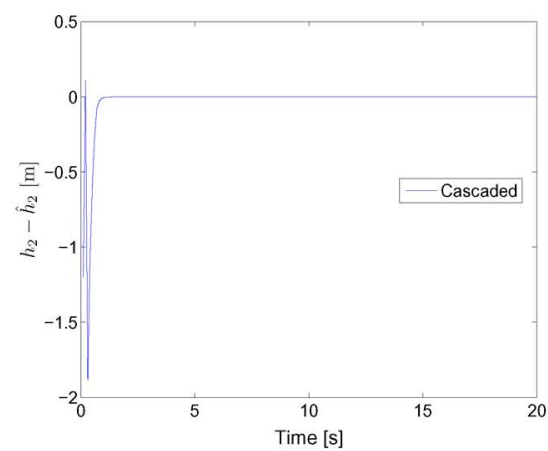

(b)

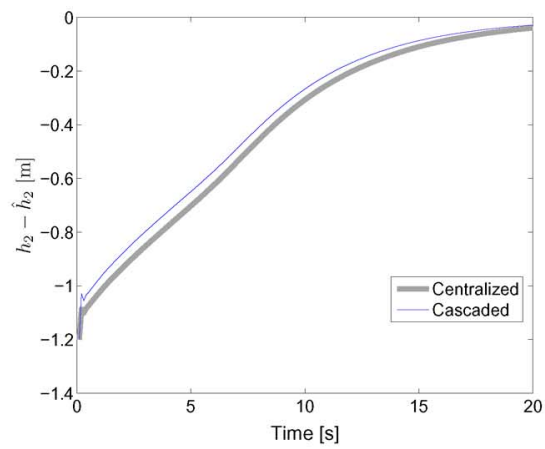

(e)

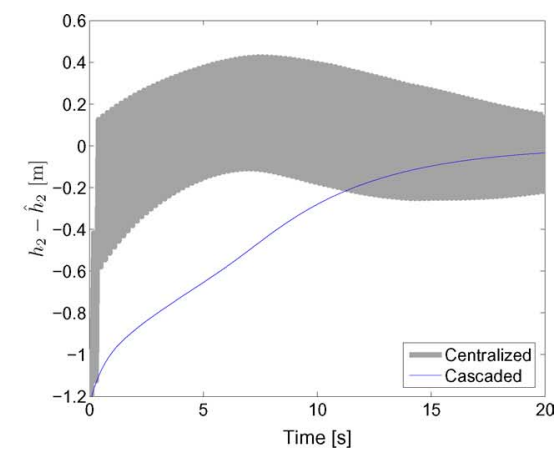

(c)

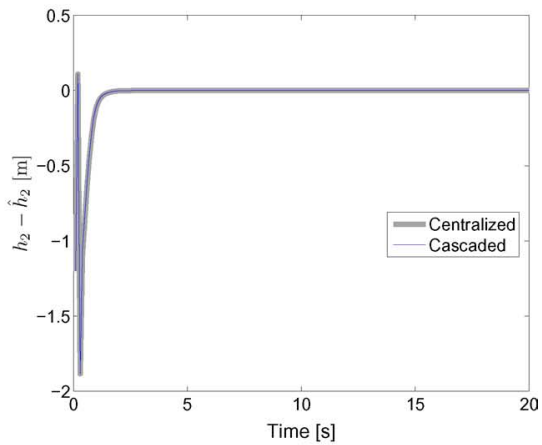

(f)

Fig. 7. Estimation errors for $h_{2}$ using centralized and cascaded observers. (a) Case 1. (b) Case 2. (c) Case 3. (d) Case 4. (e) Case 5. (f) Case 6. 
To simulate the system, the differential equations were discretized with the Euler method, using a sampling period $T=0.1 \mathrm{~s}$. The input was randomly generated, and so were the "true" and "estimated" initial states. For the presented cases, the true initial conditions were $\left[\begin{array}{lllll}1.7 & 0.4 & 0.1 & 0.4\end{array}\right]^{T}$, while the estimated ones were $\left[\begin{array}{llll}1.5 & 0.2 & 1.3 & 0.8\end{array}\right]^{T}$. The observers were designed using different LMI regions. The regions and the CPU time needed to solve the LMIs for these regions using the Yalmip toolbox [34] are presented in Table II for the centralized and cascaded observers. As can be seen, the time needed to solve the LMIs for the centralized observer is, in most cases, more than ten times larger than the time needed for the cascaded observer. This is due to the fact that for the centralized system, sixty-four $4 \times 4$ LMIs need to be solved, while for the cascaded approach, this number is reduced to $2 \times 4$ LMIs of dimension 2 .

The estimation errors of $F_{\text {in }}$ and $h_{2}$, when using centralized and cascaded observers, for the six cases are presented in Figs. 6 and 7 , respectively.

If the LMI region is the entire left half-plane, the cascaded observer converges much faster than the centralized [see Figs. 6(a) and 7(a)]. If the closed-loop poles are restricted to the interval $[-10,-2]$, but there is no restriction on $\theta$ (case 2 ), the imaginary parts of the closed-loop poles of the centralized observer are of the order $10^{6}$ so that the observer effectively becomes unusable. Therefore, only the results obtained by the cascaded setting are presented [see Figs. 6(b) and 7(b)]. If $\theta$ is constrained, the performance of the observers is comparable. For no constraints on the poles' real part (i.e., no vertical strip in Fig. 3), the estimation error on $h_{2}$ of the cascaded observer converges faster [see Fig. 7(c) and (e)]. When both the real part and the damping are constrained, the overshoot of the cascaded observer is slightly larger than that of the centralized one [see Fig. 6(d) and (f)].

\section{CONCLUSION}

In many real-life applications, a complex process model can be decomposed into simpler, cascaded subsystems. This partitioning of a process leads to increased modularity and reduced complexity of the problem, while also making the analysis easier. In this paper, we have studied the stability of a cascaded fuzzy system, based on its individual subsystems. We have proven that the stability of the individual subsystems implies the stability of the global fuzzy system. Furthermore, the proposed approach relaxes the conventional stability conditions and reduces the dimension of the LMI problem to be solved.

We have also extended the cascaded setting to state estimation. If a complex process model can be decomposed into a cascade of simpler subsystems, observers can be designed for these individual subsystems. This partitioning of a process and observer leads to increased modularity and reduced complexity of the problem, with reduced computational costs. The benefits of studying stability based on subsystems have been demonstrated in simulation examples.

In our future research, we will investigate the theoretical conditions under which fuzzy observers can be used for distributed, but not necessarily cascaded, systems.

\section{REFERENCES}

[1] T. Takagi and M. Sugeno, "Fuzzy identification of systems and its applications to modeling and control," IEEE Trans. Syst., Man, Cybern., vol. SMC-15, no. 1, pp. 116-132, Jan. 1985.

[2] C. Fantuzzi and R. Rovatti, "On the approximation capabilities of the homogeneous Takagi-Sugeno model," in Proc. 5th IEEE Int. Conf. Fuzzy Syst., pp. 1067-1072, 1996.

[3] K. Tanaka, T. Ikeda, and H. Wang, "Fuzzy regulators and fuzzy observers: Relaxed stability conditions and LMI-based designs," IEEE Trans. Fuzzy Syst., vol. 6, no. 2, pp. 250-265, May 1998.

[4] M. Johansson, A. Rantzer, and K. Arzen, "Piecewise quadratic stability of fuzzy systems," IEEE Trans. Fuzzy Syst., vol. 7, no. 6, pp. 713-722, Dec. 1999.

[5] P. Bergsten, R. Palm, and D. Driankov, "Fuzzy observers," in Proc. 10th IEEE Int. Conf. Fuzzy Syst., 2001, vol. 2, pp. 700-703.

[6] G. Feng, "A survey on analysis and design of model-based fuzzy control systems," IEEE Trans. Fuzzy Syst., vol. 14, no. 5, pp. 676-697, Oct. 2006.

[7] K. Tanaka and H. Wang, "Fuzzy regulators and fuzzy observers: A linear matrix inequality approach," in Proc. 36th IEEE Conf. Decis. Control, 1997, vol. 2, pp. 1315-1320.

[8] P. Bergsten, R. Palm, and D. Driankov, "Observers for Takagi-Sugeno fuzzy systems," IEEE Trans. Syst., Man, Cybern. B, Cybern., vol. 32, no. 1, pp. 114-121, Feb. 2002.

[9] R. Palm and P. Bergsten, "Sliding mode observer for a Takagi-Sugeno fuzzy system," in Proc. 9th IEEE Int. Conf. Fuzzy Syst., vol. 2, pp. 665670, 2000.

[10] R. Vadigepalli and F. J. Doyle, "Structural analysis of large-scale systems for distributed state estimation and control applications," Control Eng. Pract., vol. 11, pp. 895-905, 2003.

[11] R. Vadigepalli and F. J. I. Doyle, "A distributed state estimation and control algorithm for plantwide processes," IEEE Trans. Control Syst. Technol., vol. 11, no. 1, pp. 119-127, Jan. 2003.

[12] J. A. López-Orozco, J. M. de la Cruz, E. Besada, and P. Ruiprez, "An asynchronous, robust, and distributed multisensor fusion system for mobile robots," Int. J. Robot. Res., vol. 19, no. 12, pp. 914-932, Dec. 2000.

[13] S. Roumeliotis and G. Bekey, "Distributed multirobot localization," IEEE Trans. Robot. Autom., vol. 18, no. 5, pp. 781-795, Oct. 2002.

[14] T. Schmitt, R. Hanek, M. Beetz, S. Buck, and B. Radig, "Cooperative probabilistic state estimation for vision-based autonomous mobile robots," IEEE Trans. Robot. Autom., vol. 18, no. 5, pp. 670-684, Oct. 2002.

[15] H. Durrant-Whyte, B. Rao, and H. Hu, "Toward a fully decentralized architecture for multi-sensor data fusion," in Proc. IEEE Int. Conf. Robot. Autom., 1990, vol. 2, pp. 1331-1336.

[16] M. Bolic, P. M. Djuric, and S. Hong, "Resampling algorithms and architectures for distributed particle filters," IEEE Trans. Signal Process., vol. 53, no. 7, pp. 2442-2450, Jul. 2004.

[17] M. Coates, "Distributed particle filters for sensor networks," in Proc. IPSN 2004, pp. 99-107.

[18] A. Loria and E. Panteley, "Cascaded nonlinear time-varying systems: Analysis and design," in Advanced Topics in Control Systems Theory. Berlin, Germany: Springer-Verlag, 2005, pp. 23-64.

[19] M. Arcak, D. Angeli, and E. Sontag, "A unifying integral ISS framework for stability of nonlinear cascades," SIAM J. Control Optim., vol. 40, pp. 1888-1904, 2002.

[20] E. D. Sontag, "Smooth stabilization implies coprime factorization," IEEE Trans. Autom. Control, vol. 34, no. 4, pp. 435-443, Apr. 1989.

[21] P. Seibert and R. Suarez, "Global stabilization of nonlinear cascade systems," Syst. Control Lett., vol. 14, pp. 347-352, 1990.

[22] E. Sontag, "Remarks on stabilization and input-to-state stability," in Proc. IEEE Conf. Decis. Control, 1989, pp. 1376-1378.

[23] M. Jankovic, R. Sepulchre, and P. Kokotovic, "Constructive Lyapunov stabilization of nonlinear cascade systems," IEEE Trans. Autom. Control, vol. 41, no. 12, pp. 1723-1735, Dec. 1996.

[24] A. Chaillet and A. Loria, "Necessary and sufficient conditions for uniform semiglobal practical asymptotic stability: Application to cascaded systems," Automatica, vol. 42, pp. 1899-1906, 2006.

[25] A. Bacciotti, P. Boieri, and L. Mazzi, "Linear stabilization of nonlinear cascade systems," Math. Control, Signals, Syst., vol. 6, pp. 146-165, 1993.

[26] K. Roebenack and A. Lynch, "Observer design using a partial nonlinear observer canonical form," Int. J. Appl. Math. Comput. Sci., vol. 16, pp. 333-343, 2006.

[27] P. Bergsten, "Observers and controllers for Takagi-Sugeno fuzzy systems," Ph.D. dissertation. Örebro Univ., Örebro, Sweden, 2001. 
[28] A. Rantzer and M. Johansson, "Piecewise linear quadratic optimal control," IEEE Trans. Autom. Control, vol. 45, no. 4, pp. 629-637, Apr. 2000.

[29] M. Johansson and A. Rantzer, "Computation of piecewise quadratic Lyapunov functions for hybrid systems," IEEE Trans. Autom. Control, vol. 43, no. 4, pp. 555-559, Apr. 1998.

[30] M. Johansson, "Piecewise linear control systems," Ph.D. dissertation, Dept. Autom. Control, Lund Inst. Technol., Lund, Sweden, 1999.

[31] W.-J. Wang and C.-H. Sun, "Relaxed stability and stabilization conditions for a TS fuzzy discrete system," Fuzzy Sets Syst., vol. 156, pp. 208-225, 2005.

[32] A. Baddou, M. Naib, F. Tadeo, and A. Benzaouia, "On improving the convergence rate of linear continuous-time systems subject to asymmetrically constrained control," Math. Problems Eng., vol. 2006, pp. 894291-89429-14, 2006.

[33] M. Chilali and P. Gahinet, " $H_{\infty}$ design with pole placement constraints: An LMI approach," IEEE Trans. Autom. Control, vol. 41, no. 3, pp. 358367, Mar. 1996.

[34] J. Löfberg, "YALMIP: A toolbox for modeling and optimization in MATLAB," presented at the CACSD Conf., Taipei, Taiwan, 2004.

[35] N. Waurajitti, J. Ngamwiwit, and Y. Prempraneerach, "Adaptive fuzzy sliding mode controller for two cascaded tanks level control," in Proc. TENCON, vol. 2, pp. 592-597, 2000.

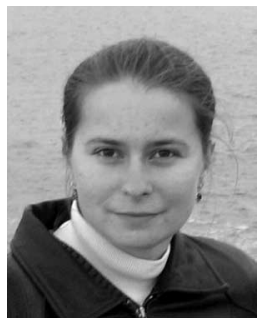

decentralized systems.

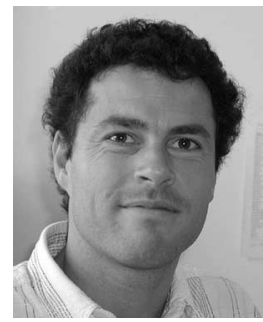

Robert Babuška received the M.Sc. degree in control engineering from the Czech Technical University, Prague, Czech Republic, in 1990 and the Ph.D. degree from Delft University of Technology, Delft, The Netherlands, in 1997.

He has had faculty appointments at the Technical Cybernetics Department, Czech Technical University, and at the Electrical Engineering Faculty, Delft University of Technology. He is currently a Professor at the Delft Center for Systems and Control, Faculty of Mechanical Engineering, Delft University of Technology. His current research interests include neural and fuzzy systems for modeling and identification, fault-tolerant control, learning and adaptive control, and dynamic multiagent systems. He is involved in several projects in which these techniques are being applied in the fields of mechatronics, robotics, and aerospace. He has authored or coauthored over 190 publications, including one research monograph (Kluwer Academic), two edited books, 24 invited chapters in books, 48 journal papers, and more than 120 conference papers. He is an Associate Editor of Engineering Applications of Artificial Intelligence and an Area Editor of Fuzzy Sets and Systems.

Prof. Babuška served as an Associate Editor of the IEEE TRANSACTIONS ON FUZZY SYSTEMS and as the Chairman of the International Federation of Automatic Control Technical Committee on Cognition and Control.

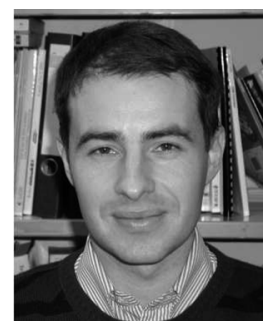

Bart De Schutter (M'08) received the M.Sc. degree in electrotechnical-mechanical engineering and the Ph.D. degree (summa cum laude with congratulations of the examination jury) in applied sciences from Katholieke Universiteit Leuven (K.U. Leuven), Leuven, Belgium, in 1991 and 1996, respectively.

He is currently a Full Professor at the Delft Center for Systems and Control and also at the Marine and Transport Technology Department, Delft University of Technology, Delft, The Netherlands. His current research interests include hybrid systems control, multiagent systems, control of intelligent transportation systems, and optimization.

Prof. De Schutter was awarded the 1998 Society for Industrial and Applied Mathematics (SIAM) Richard C. DiPrima Prize and the 1999 K.U. Leuven Robert Stock Prize for his Ph.D. thesis. 